\title{
Stability of Hypersonic Boundary Layers on a Cone at an Angle of Attack
}

\author{
P. Balakumar ${ }^{*}$ and Lewis R. Owens ${ }^{\dagger}$ \\ Flow Physics and Control Branch NASA Langley Research Center, Hampton, VA 23681
}

\begin{abstract}
The stability and receptivity of a three-dimensional hypersonic boundary layer over a $7^{\circ}$ half-angle straight cone at an angle of attack of $6^{\circ}$ is numerically investigated at a freestream Mach number of 6.0 and a Reynolds number of $10.4 * 10 \% \mathrm{~m}$. The generation and evolution of stationary crossflow vortices are also investigated by performing simulations with three-dimensional roughness elements located on the surface of the cone. The flow fields with and without the roughness elements are obtained by solving the full NavierStokes equations in cylindrical coordinates using a fifth-order accurate weighted essentially non-oscillatory (WENO) scheme for spatial discretization and a third-order total-variationdiminishing (TVD) Runge-Kutta scheme for temporal integration. Stability computations produced azimuthal wavenumbers in the range of $\boldsymbol{m} \sim \mathbf{2 0 - 5 0}$ for the most amplified traveling disturbances and in the range of $m \sim 30-70$ for the stationary disturbances. The frequency of the unstable second-mode ranges from $400 \mathrm{kHz}$ to $900 \mathrm{kHz}$ along the windward ray. The $\mathrm{N}$ Factor computations predicted transition would occur more forward on the sides of the cone as compared to the transition fronts near the windward and the leeward rays. The simulations also show the crossflow vortices originating from the nose region propagate towards the leeward ray. No perturbations were observed toward the windward half of the cone.
\end{abstract}

\section{Introduction}

$\mathbf{T}$ RANSITION in two-dimensional hypersonic boundary layers in quiet environments is generally caused by second-mode (Mack's mode) ${ }^{1}$ disturbances. These modes originate due to the appearance of a supersonic region relative to the disturbance phase velocity. Numerical, experimental, and theoretical investigations have been performed to understand these instability modes for hypersonic boundary layers. ${ }^{1-9}$ These investigators found the most amplified second-mode disturbances are two-dimensional. The phase velocity of first-mode disturbances varies from 1-1/M to $C_{s}$, where $M$ is the free-stream Mach number and $C_{s}$ is the mean velocity at which the generalized inflection of the boundary-layer profile occurs. For the second mode, the phase velocity varies from $C_{s}$ to 1 . Acoustic disturbances with the phase velocity $1-1 / M(U-a$, slow wave) are very efficient in exciting the first-mode instabilities in hypersonic boundary layers because they synchronize with the phase velocity. In previous investigations ${ }^{10-14}$, the first author of this paper along with various co-authors investigated the interactions of slow and fast acoustic waves with hypersonic boundary layers over sharp and blunt flat plates, wedges, and cones. They found that flows over geometries with sharp leading edges or tips produce instability waves that originate very close to the leading edge or tip and have receptivity coefficients about 5 to 10 times the amplitude of the forced slow acoustic wave. They also showed that the amplitude of the instability waves generated by the slow acoustic waves is about 20 times larger than that for fast acoustic waves. In addition, they observed that bluntness and wall cooling stabilize the first-mode disturbances and the initial amplitudes of the instability waves are several orders of magnitude smaller than the amplitude of the forced acoustic waves.

In this paper, we shift our focus to the stability of three-dimensional hypersonic boundary layers. Several experiments were performed in the past to understand the stability and transition of supersonic and hypersonic twoand three-dimensional boundary layers. These experiments can be divided into two groups. One group includes

\footnotetext{
${ }^{*}$ Research Scientist. Corresponding author. Tel: 757 864-8453. E-mail: ponnampalam.balakumar-1@nasa.gov.

${ }^{\dagger}$ Research Scientist, M.S. 170.
} 
experiments where the transition onsets are measured at various flow conditions. ${ }^{15-19}$ In these experiments, the effects of bluntness and angle of attack on the transition front were investigated. The second group of experiments includes stability experiments where the stability characteristics of the boundary layers were investigated. ${ }^{2,20-32}$ Stetson and his co-workers systematically investigated the stability characteristics of hypersonic boundary layers in conventional tunnels. ${ }^{3,20-24}$ Experiments were performed on sharp and blunt cones with a $7^{\circ}$ half angle at a freestream Mach number of 8 . These cone models were tested at zero and nonzero angles of attack and with adiabatic and cooled surface temperature conditions. Several experiments were also performed in the NASA Langley Research Center Mach 6 Quiet Tunnel. ${ }^{25-27}$ These experiments were performed on a $5^{\circ}$ half-angle straight cone and over a flared cone for various test conditions. Recently, experiments were performed at the Purdue Boeing/AFSOR Mach-6 Quiet Tunnel (BAM6QT) on a $7^{\circ}$ half-angle cone at zero and nonzero angles of attack ${ }^{28-31}$ and at the Hypersonic Ludwieg Tube Braunschweig (HLB) facility. ${ }^{32}$ All of the experiments referenced here agree with the theoretical predictions, which suggest that transition in hypersonic boundary layers is dominated by the high frequency second-mode-type disturbances. In addition, they observed that adding a small amount of nose-tip bluntness increases the transition Reynolds number. However, they also found that further increases in the bluntness reverse this trend and the transition onset moves upstream. The reason for the reversal of the transition onset movement is not yet understood. And finally, they also found that in flows over cones at angle of attack the boundary layer along the windward direction becomes more stable and on the leeward side it becomes less stable as compared to that obtained at zero angle of attack.

The linear instability of axisymmetric three-dimensional compressible boundary layers for a rotating cone was numerically investigated by Balakumar and Reed. ${ }^{33}$ Their calculations show that due to the presence of crossflow, the growth rate of traveling disturbances increases by a factor of 2 to 4 when compared to a two-dimensional flow over a non-rotating cone. They also found this increasing growth rate diminishes with increasing Mach number. Furthermore, they found the growth of the second mode depends only on the velocity along the inviscid streamlines and is not altered by the crossflow that exists in the direction perpendicular to the inviscid streamlines. Hence, one expects to see both unstable stationary and traveling crossflow disturbances as well as unstable second-mode disturbances in hypersonic three-dimensional boundary layers.

Recently, we studied the stability of a three-dimensional supersonic boundary layer over a $7^{\circ}$ half-angle cone at a nonzero angle of attack. ${ }^{34}$ This investigation shows the stability characteristics of flows over cones at angle of attack can be divided into three general regions. One region is close to the windward symmetry plane, where the crossflow is weak and transition occurs due to the unstable first modes. The second is the region near the leeward plane, where the crossflow is again weak but the velocity profiles become highly inflectional due to the accumulation of low speed fluid from either side of the symmetry plane. This region becomes much more unstable and the transition occurs much earlier than along the windward plane. Third is the larger middle region, where the crossflow is strong and the transition occurs due to traveling and/or stationary crossflow disturbances. The crossflow gradually increases from the windward plane with increasing azimuthal angle, reaches its peak around $120^{\circ}$ and then decreases as the azimuthal angle continues toward the leeward plane. This crossflow character is reflected in the computed transition fronts as the transition front moves upstream with increasing azimuthal angle up to $120^{\circ}$ and then moves downstream again with further increase in the azimuthal angle.

The objective of the present investigation is to numerically study the linear instability, transition onset, receptivity, and the growth of stationary crossflow vortices in hypersonic boundary layers over a sharp cone at a given angle of attack. The conditions for this study include a hypersonic flow of $M=6.0$ over a $7^{\circ}$ half-angle sharp cone at an angle of attack of $6^{\circ}$ (Fig. 1). The ratio of the angle of attack to the cone half angle is approximately 0.86 , which is higher than what has been typically studied in previous investigations. The nose radius is $0.05 \mathrm{~mm}(0.002$ inches). These parameters are selected to validate the stability experiments that are being concurrently performed in the Purdue BAM6QT. ${ }^{28-31}$ The numerical simulations are performed by solving the three-dimensional Navier-Stokes equations in cylindrical coordinates using a fifth-order accurate weighted essentially non-oscillatory (WENO) scheme for spatial discretization and a third-order total-variation-diminishing (TVD) Runge-Kutta scheme for temporal integration. The stability computations are performed using the local parallel theory and the N-Factor computations are performed using the three-dimensional transition prediction code, eMalik $3 d .^{35}$ The receptivity analysis is carried out by performing simulations with three-dimensional roughness elements placed: (1) in a ring around the cone at a fixed distance from the apex, and (2) along a ray spaced at a constant wavelength in the axial direction (Fig. 1). Computations are performed for a unit Reynolds number of $10.4 * 10^{6} / \mathrm{m}$, which matches the experimental condition. The governing equations, solution algorithm, computational grid, flow conditions and roughness descriptions are discussed in section II. Mean flow profiles, linear stability results, transition onsets, 
receptivity and the evolution of crossflow vortices originating from the roughness elements are presented in section III. Conclusions are drawn in section IV.

\section{Computational Approach}

\section{A. Governing Equations}

The equations solved are the three-dimensional unsteady compressible Navier-Stokes equations in conservation form in cylindrical coordinates

$$
\begin{gathered}
\frac{\partial}{\partial t} r Q+\frac{\partial r F}{\partial x}+\frac{\partial r G}{\partial r}+\frac{\partial H}{\partial \theta}=S . \\
Q=\left[\begin{array}{l}
\rho \\
\rho u \\
\rho v \\
\rho w \\
\rho E
\end{array}\right]
\end{gathered}
$$

Here $(x, r, \theta)$ are the cylindrical coordinates, $(u, v, w)$ are the velocity components, $\rho$ is the density, and $p$ is the pressure. $E$ is the total energy given by

$$
\begin{gathered}
E=e+\frac{u^{2}+v^{2}+w^{2}}{2}, \\
e=c_{v} T, p=\rho R T .
\end{gathered}
$$

Here $e$ is the internal energy and $T$ is the temperature. The fluxes $F, G, H$, and the source term $S$ are described in Ref. 36. The viscosity $(\mu)$ is computed using Sutherland's law and the coefficient of conductivity $(k)$ is given in terms of the Prandtl number $P r$. The variables $\rho, p, T$ and velocity are non-dimensionalized by their corresponding reference variables $\rho_{\infty}, p_{\infty}, T_{\infty}$ and $\sqrt{R T_{\infty}}$, respectively. The reference value for length is computed by $\sqrt{v x_{0} / U_{\infty}}$, where $x_{0}$ is a reference location. For the computation, the equations are transformed from the physical coordinate system $(x, r, \theta)$ to the computational curvilinear coordinate system $(\xi, \eta, \zeta)$ in a conservative manner.

\section{B. Solution Algorithm}

The governing equations are solved using a fifth-order accurate weighted essentially non-oscillatory (WENO) scheme for space discretization and using a third-order, total variation diminishing (TVD) Runge-Kutta scheme for time integration. These methods are suitable in flows with discontinuities or high gradient regions. The governing equations are solved discretely in a uniform structured computational domain where flow properties are known point-wise at the grid nodes. In a given direction, the spatial derivatives are approximated to a higher order at the nodes, using the neighboring nodal values in that direction. The resulting equations are then integrated in time to get the point values as a function of time. Since the spatial derivatives are independent of the coordinate directions, multi dimensions can be easily added to the method. It is well known that approximating a discontinuous function by a higher-order (two or more) polynomial generally introduces oscillatory behavior near the discontinuity, and this oscillation increases with the order of the approximation. The essentially non-oscillatory (ENO) method and its successor the WENO method were developed to maintain the higher-order approximations in the smooth regions and to eliminate or suppress the oscillatory behavior near the discontinuities. These methods systematically adopt or select the stencils based on the smoothness of the function that is being approximated. The WENO and the TVD 
methods and the formulas are explained in Ref. 37. The application of the ENO method to the N-S equations is shown in Ref. 38. The solution method implemented in this computation is described in detail in Ref. 39.

At the outflow boundary, extrapolation boundary conditions are used. At the wall, viscous conditions are used for the velocities and a constant temperature condition is employed for the temperature. The density at the wall is computed from the continuity equation. In the azimuthal direction, symmetry conditions are imposed at the $\theta=0^{\circ}$ and $\theta=180^{\circ}$ boundaries. The free-stream values are prescribed at the upper boundary that lies outside the bow shock. The steady-state computations are performed using a variable time step until the maximum residual reaches a small value, $\sim 10^{-10}$.

\section{Computational Grid}

The nose region of the cone is modeled as a circle of the form

$$
\left(x-r_{0}\right)^{2}+y^{2}=r_{0}^{2}
$$

Here $r_{0}$ is the radius of the tip bluntness. The circular nose is smoothly merged with the cone by a tenth-order polynomial. Simulations are performed for a nose radius of $r_{0}=0.05 \mathrm{~mm}(0.002 \mathrm{in}$.). The length of the cone is 40 $\mathrm{cm}$.

The grid stretches in the $\eta$ direction close to the wall and is uniform outside the boundary layer. In the $\xi$ direction, the grid is symmetric about the tip and very fine near the nose and is uniform in the flat region. The grid is uniform in the azimuthal direction. The far-field boundary outside of the shock follows a parabola to capture the boundary layer accurately. Calculations were performed using a grid size of $(3001 * 251 * 361)$. Due to the very fine grid distributions in the azimuthal and axial directions near the nose, the allowable CFL number is limited to very small values and it becomes very expensive to compute the entire domain at once. To overcome this, calculations are performed in several steps. First, the computations are done near the nose region with a small CFL number of 0.01 . Second, the flow properties in the middle of this domain are fed as inflow conditions for the next domain and the computations are carried out with a larger CFL number of 0.50 .

\section{Flow Conditions}

The computations are performed for a hypersonic flow with a Mach number of $M=6.0$ over a $7^{\circ}$ half-angle cone at an angle of attack of $6.0^{\circ}$. The flow parameters are given in Table 1 and a schematic diagram of the computational set up is shown in Fig. 1.

\section{Table 1. Flow parameters}

Free-stream Mach number: $M_{\infty}=6.0$

Free-stream Reynolds numbers: $\operatorname{Re}_{\infty}=10.4 * 10^{6} / \mathrm{m}$.

Free-stream temperature: $T_{\infty}=53.42^{\circ} \mathrm{K}$

Stagnation pressure: $\mathrm{P}_{0}=965.3 \mathrm{kPa}(140 \mathrm{psi})$

Wall temperature: $T=300.0^{\circ} \mathrm{K}$

Prandtl number: $P r=0.72$

Ratio of specific heats: $\gamma=1.4$

Local Reynolds number: $\sqrt{\operatorname{Re}_{x}}=\sqrt{\frac{U_{e} x}{v}}$

\section{E. Roughness Definition}

Two types of roughness patterns are used in the simulations to investigate the generation and the evolution of crossflow vortices in three-dimensional supersonic boundary layers over a cone at an angle of attack.

\section{Roughness 1}

The first type consists of three-dimensional roughness elements that are periodic in the azimuthal direction placed on the surface of the cone at a fixed axial location close to the nose-tip region. The shape of the roughness is of the form:

$$
y_{c}(x, \theta)=h e^{-\sigma_{x}\left(\frac{x-x_{r}}{l}\right)^{2}} \cos m \theta
$$


Here $y_{c}$ is the height of the roughness normal to the surface of the cone, $h$ is the maximum height, $x_{r}$ is the axial location of the roughness, $m$ is the azimuthal wavenumber, $l$ is the length scale and $\sigma_{x}$ is a constant that determines the width of the roughness in the axial direction. Computations are performed for $m=40$ and for the values $x_{r}, h$, and $\sigma$ that are given in Table 2. The Fourier transform of the roughness shape is given by

$$
\begin{aligned}
\tilde{y}_{c}\left(k_{x}\right) & =\frac{1}{2 \pi} \int_{-\infty}^{\infty} y_{c}(x) e^{-i k_{x} x} d x \\
& =\bar{h} \frac{1}{2 \sqrt{\pi \sigma_{x}}} e^{-\frac{k_{x}^{2}}{4 \sigma_{x}}}
\end{aligned}
$$

Here the variables $k_{x}$ and $h$ are made dimensionless by the length scale $l$.

Table 2. Location and the height of the roughness 1

\begin{tabular}{|c|c|c|c|c|}
\hline $\begin{array}{c}\mathbf{x}_{\mathbf{r}} \\
(\mathrm{mm})\end{array}$ & $\begin{array}{c}\mathbf{h} \\
(\mathrm{mm})\end{array}$ & $\begin{array}{c}\mathbf{l} \\
(\mathrm{mm})\end{array}$ & $\sigma_{\mathbf{x}}$ & $\mathbf{h} / \boldsymbol{\delta}$ \\
\hline 50 & 0.01 & 1.0 & 1 & $1 / 20 \sim 1 / 40$ \\
\hline
\end{tabular}

\section{Roughness 2}

The second type consists of three-dimensional roughness elements distributed along a ray at a constant azimuthal angle $\theta_{r}$, which is closer to the windward side. The shape of the roughness is of the form

$$
\begin{aligned}
y_{c}(x, \theta) & =h e^{-\sigma_{\theta}\left(\frac{\theta-\theta_{r}}{\Delta \theta}\right)^{2}} \sum_{n=1}^{N} e^{-\sigma_{x}\left(\frac{x-x_{n}}{l}\right)^{2}} \\
x_{n} & =x_{1}+(n-1) * \lambda
\end{aligned}
$$

Here $y_{c}$ is the height of the roughness normal to the surface of the cone, $h$ is the maximum height, $\theta_{r}$ is the azimuthal location of the roughness, $l$ is the length scale and $\sigma_{\theta}, \sigma_{x}$ are constants that determine the width of the roughness in the azimuthal and in the axial directions. The axial location of the first roughness element is $x_{1}$ and $\lambda$ is the distance between the roughness elements. Computations are performed for $x_{1}=15 \mathrm{~mm}, \theta_{r}=25 \mathrm{deg}, \lambda=10 \mathrm{~mm}$ and for the values $h$, and $\sigma_{x}, \sigma_{\theta}$ that are given in Table 3 .

Table 3. Location and the height of the roughness 2

\begin{tabular}{|c|c|c|c|c|c|c|}
\hline $\begin{array}{c}\theta_{\mathrm{r}} \\
(\mathrm{deg})\end{array}$ & $\begin{array}{c}\mathbf{h} \\
(\mathrm{mm})\end{array}$ & $\sigma_{\theta}$ & $\mathbf{h} / \delta$ & $\begin{array}{c}\Delta \theta \\
(\mathrm{deg})\end{array}$ & $\begin{array}{c}\mathbf{x}_{1} \\
(\mathbf{m m})\end{array}$ & $\begin{array}{c}\lambda \\
(\mathbf{m m})\end{array}$ \\
\hline 25 & 0.01 & 2 & $1 / 12$ & 2.5 & 15 & 10 \\
\hline
\end{tabular}

\section{Results}

\section{A. Mean flow without roughness}

The mean flow density contours and the boundary-layer profiles computed using the WENO code without the roughness elements are given in Figs. 2-7. The density contours in the larger domain are shown in Fig. 2(a) while the flow field near the nose tip region is shown in Fig. 2(b). We see a stronger shock along the windward plane and a weaker one along the leeward plane. The shock angles are $25.7^{\circ}$ and $31.4^{\circ}$ along the windward and the leeward symmetry planes, respectively. The shock is detached about $0.02 \mathrm{~mm}$ from the nose of radius $0.05 \mathrm{~mm}$. The density contours in cross-sectional planes for two different axial locations, $x=9$ and $330 \mathrm{~mm}$ are shown in Fig. 3. The 
$40^{\text {th }}$ Fluid Dynamics Conference, June 28-July $1^{\text {st }}, 2010$, Chicago, Illinois

accumulation of low momentum flow produces a thickening of the boundary layer approaching the leeward plane. Very close to the tip, the boundary-layer thickness increases gradually up to the leeward side. Further downstream, bulges start to form in a narrow region near the leeward plane. This accumulation of low-speed fluid is advected towards the leeward plane by the crossflow.

The boundary-layer density profiles at three azimuthal planes, $\theta=0^{\circ}$ (windward), $\theta=90^{\circ}$ (side), and $\theta=180^{\circ}$ (leeward) are shown in Figs. 4-6. The profiles are displayed for the axial stations $x=10,50,100,200$ and $300 \mathrm{~mm}$ downstream of the nose tip. The profiles including the shock in the physical coordinate (shown in $\mathrm{mm}$ ) are given in Figs. 4-6(a), while a close-up of the boundary-layer region is presented in Figs. 4-6(b). Figures 4-6 (c) show the boundary-layer profiles plotted in the similarity coordinate. Note the vertical scales increase in range in these figures from Fig. 4 to Fig. 6 due to the increasing boundary-layer thickness in the azimuthal direction. The boundary-layer thicknesses at $x=100 \mathrm{~mm}$ at the $\theta=0^{\circ}$ (windward), $\theta=90^{\circ}$ (side), and $\theta=180^{\circ}$ (leeward) locations are $0.50,0.70$ and $5.0 \mathrm{~mm}$, respectively. The boundary layer along the cone ray in the leeward plane is about 10 times thicker than the ray in the windward plane.

It is known that the inviscid flow over a sharp cone at an angle of attack is conical in the azimuthal direction, which means the flow properties remain constant along each ray emanating from the apex. This flow behavior allows for similarity solutions along the rays. Hence, the boundary-layer equations for flow over a sharp cone at an angle of attack can be transformed into simplified partial differential equations that are solved by marching in the azimuthal direction. The similarity equations are given in Ref. 40. It is interesting to see from Figs. 4(c) and 5(c) that the velocity profiles plotted in similarity coordinates exhibit the expected collapsing behavior. The boundary-layer thicknesses in the similarity variable are about 6.0 and 8.0 along the $\theta=0^{\circ}, \theta=90^{\circ}$ rays. As we approach the leeward plane the parabolic assumption in the azimuthal direction breaks down and the similarity behavior is lost as shown in Fig. 6(c).

Figures $7(\mathrm{a}), 7(\mathrm{~b})$ and $7(\mathrm{c})$ include plots of the boundary-layer velocity profiles along the inviscid streamlines $\left(U_{s}\right)$, along the azimuthal direction $(W)$ and along the crossflow directions $\left(U_{c}\right)$ at an axial station of $x=200 \mathrm{~mm}$. The profiles are plotted for various azimuthal angles ranging from $\theta=0^{\circ}$ to $180^{\circ}$. The azimuthal velocity profiles presented in Fig. 7(b) show the boundary layer separating close to the leeward side at an angle of $\theta=165^{\circ}$. The separation exists at upstream axial locations too. The separation bubble starts to appear along the leeward ray from $\mathrm{x}$ $\sim 12 \mathrm{~mm}$. The boundary-layer stream-wise velocity profiles vary slowly up to $165^{\circ}$ and then increase steeply in the last $15^{\circ}$ near the leeward plane. Notice that in Fig. 7(a) the boundary-layer profile along the leeward ray, $\theta=180^{\circ}$ is inflectional. In Fig. 7(c), the crossflow velocity increases slowly from the windward plane with increasing azimuthal angle, reaches the highest magnitude of 0.14 around $120^{\circ}$ and then decreases slowly towards the leeward plane. Also note that substantial crossflow exists even at $\theta=165^{\circ}$ from the windward plane. The contours of the computed crossflow Reynolds numbers for the unit Reynolds number of $10.4 * 10^{6} / \mathrm{m}$ are shown in Fig. 8. The maximum crossflow Reynolds numbers reach about 2600 toward the upper end of the cone.

\section{B. Linear stability and the transition onset}

The linear stability results obtained from the local parallel theory at given axial stations, $x=100,200$, and 300 $\mathrm{mm}$, are plotted in Fig. 9. At each axial station $x$, the results show the growth rates as a function of frequency for selected azimuthal stations, $\theta=0^{\circ}, 30^{\circ}, 60^{\circ}, 90^{\circ}, 120^{\circ}$ and $180^{\circ}$. The solid lines represent the second-mode disturbances with the azimuthal wavenumber $m=0$ and the dotted lines represent the unstable crossflow disturbances with the wavenumber $m=40 \sim 60$ corresponding to the most amplified wave. First, notice that the unstable second-mode frequencies decrease as the azimuthal angle increases. This change is due to the growth of the boundary layer in the azimuthal direction toward the leeward plane. At $x=200 \mathrm{~mm}$, the unstable frequencies of the second mode decrease from a range of 550 to $650 \mathrm{kHz}$ at $\theta=0^{\circ}$ (windward plane) to a range of 125 to $200 \mathrm{kHz}$ at $\theta$ $=150^{\circ}$ (near the leeward plane). However, the maximum growth rates remains about the same. The unstable frequencies of the three-dimensional traveling crossflow and the first-mode disturbances are similar at each of the selected azimuthal locations covering a range of about 0 to $200 \mathrm{kHz}$. The maximum growth rates occur between $60^{\circ}$ and $150^{\circ}$ rays. The most amplified frequencies are in the range of $0 \sim 60 \mathrm{kHz}$. The growth rates for the stationary disturbances along a given cone ray are smaller than the maximum growth rates for the corresponding traveling disturbances. The growth rates for the stationary disturbances at $\theta=90^{\circ}$ are $0.0052,0.0061$, and 0.0066 at $x=100$, 200 and $300 \mathrm{~mm}$ and the maximum growth rates for the traveling disturbances are $0.0059,0.0071$ and 0.0077 at the same locations. The differences in growth rates for the stationary and traveling disturbances increases in the region closer to the windward side $\left(\theta=0^{\circ}, 30^{\circ}, 60^{\circ}\right)$ and decreases in the region closer to the leeward side $\left(\theta=120^{\circ}, 150^{\circ}\right)$.

The N-Factor traces obtained from the eMalik3d $\operatorname{code}^{35}$ using the envelope method for the traveling disturbances are shown in Fig. 10(a). Each trace is obtained by starting the computations near the neutral point for a fixed 
$40^{\text {th }}$ Fluid Dynamics Conference, June 28-July $1^{\text {st }}, 2010$, Chicago, Illinois

frequency and then marching downstream along the streamline direction while seeking the maximum growth rate at each station. Each trace ends at the location where $N=11$. The figure shows the results for the frequencies that reached the largest N-Factor at that location. The unstable frequency for this case is about $50 \mathrm{kHz}$. The transition front takes on an arc shape with the transition front moving far forward in the crossflow region and further downstream near the windward and leeward regions. The earliest detection of transition occurred along the $110^{\circ}$ ray at the location $x=0.20 \mathrm{~m}$. The predicted transition near the leeward ray is about $x=0.30 \mathrm{~m}$ and the transition along the $30^{\circ}$ ray is at $x=0.40 \mathrm{~m}$. Comparison with Fig. 8 shows that the early transition happens in the region where crossflow Reynolds number is high $(>\sim 1800)$. The traces for the stationary disturbances are presented in Fig. 10(b). The N-Factors are smaller than 11 for this case within the computational domain except within the rays $90^{\circ}$ and $160^{\circ}$. The highest N-Factors reached are noted at the end of the traces in the figure.

\section{The generation and the evolution of stationary crossflow vortices}

The generation and evolution of stationary crossflow vortices in a hypersonic boundary layer at angle of attack were simulated by placing isolated roughness elements on the surface of the cone (recall Fig. 1). The parameters of the roughness 1 elements are given in Table 2 . The roughness 1 pattern is placed at $x_{r}=50 \mathrm{~mm}$, the azimuthal wavenumber is $m=40$, and the height of the roughness is $h=0.01 \mathrm{~mm}$. The contours of the axial velocity, $u$, in the $(x, r \theta)$ plane are shown in Fig. 11(a). In this figure, the $u$-velocity contour is presented for a constant normal grid number of 40 . The image represents the footprints of the stationary crossflow vortices originating from each of the roughness elements. The contours clearly show the movement of the crossflow vortices toward the leeward plane.

The second roughness pattern is placed along a given cone ray, $\theta_{r}=25^{\circ}$, as described in Table 3 , with the same roughness height as the first roughness pattern. The footprints of the stationary crossflow vortices originating from each of the roughness elements of the second roughness pattern are presented in Fig. 11(b). This second roughness pattern appears to produce more crossflow vortex footprints that are more intense when compared to the first roughness pattern of Fig. 11(a). Figures 12(a) and 12(b) are shown to provide a closer look at the crossflow vortices by subtracting the mean $u$-velocity component to highlight the maximum $u$-velocity perturbations. A very regular set of vortical footprints is seen in Fig. 12(a) that can be easily traced back to the roughness element of origin in pattern 1. It is interesting to observe that the contours of constant amplitude resemble the transition front pattern observed in Fig. 10(b). The amplitudes reach peak values further upstream on the sides as compared to those near the windward and leeward planes. This agrees with the stability predictions in which the crossflow stability is more unstable in the region $\theta=60^{\circ}$ to $120^{\circ}$ than in the regions closer to the windward and leeward planes. In contrast, the corresponding footprints for roughness pattern 2 appear to be more irregular making it very difficult to identify their element of origin. However, the figure shows that perturbations seen in downstream originate from the roughness elements located in the region $x \sim 15$ to $60 \mathrm{~mm}$. There are only five roughness elements in the region $x \sim 15$ to 55 $\mathrm{mm}$. Hence, it appears that the disturbances that originate from the roughness elements split into multiple vortices as they evolve downstream. The vortices generated by the roughness elements grow and mingle and produce the less regular pattern that is visualized in Fig. 12 (b).

The contours of the axial velocity, $u$, at different cross-sectional planes, $x=100,150,200$, and $250 \mathrm{~mm}$, for the roughness pattern 1 are shown in Fig. 13. The wall-normal variation of the $u$-velocity for the azimuthal angle range of $20^{\circ} \leq \theta \leq 180^{\circ}$ is presented in Fig. 13. In this figure, the windward side corresponds to the right side of the plot and the leeward side is toward the left. As discussed previously, the vortices are confined to regions close to the leeward plane. At the last station, $x=250 \mathrm{~mm}$, the vortices are confined between the cone ray $\theta=100^{\circ}$ and the leeward plane. The figures clearly display the standard co-rotating crossflow vortices near the leeward side, which rotate towards the windward side.

The contours of the axial velocity, $u$, at different cross-sectional planes, $x=100,150,200$, and $250 \mathrm{~mm}$, for the roughness pattern 2 are shown in Fig. 14. For this pattern, there appears to be more vortices present, which are confined between the ray $\theta=60^{\circ}$ and the leeward plane. Like the vortices observed for pattern 1 , the pattern 2 vortices also display co-rotating crossflow vortices, but the rotation towards the windward side begins at a lower azimuthal angle. For pattern 1, the co-rotation toward the windward side starts at $\theta=110^{\circ}$. For pattern 2, the corotation becomes evident as low as $\theta=90^{\circ}$.

The quantitative information about the perturbations induced by the roughness patterns 1 and 2 is shown in Figs. 15-18. In figures 15 and 16 , the maximum axial velocity perturbations at five downstream locations $x=55,75,100$, 125 and $150 \mathrm{~mm}$ is presented. We only display the results up to $150 \mathrm{~mm}$. Beyond $x>150 \mathrm{~mm}$, the amplitudes reach large values and nonlinear effects start to set in. These line plots are another way to visualize the development of the crossflow vortices and potentially make it easier to see the trajectories than the related contour plots shown in Figs. 13 and 14. The perturbations immediately downstream of the roughness elements are shown in Fig. 15(a). The 
$40^{\text {th }}$ Fluid Dynamics Conference, June 28-July $1^{\text {st }}, 2010$, Chicago, Illinois

wavelengths of the disturbances are about $\Delta \theta \sim 9^{\circ}$. This agrees with the input wavenumber of $m=40$ for the roughness pattern 1. The amplitude of the perturbations has a peak value of 0.004 near $\theta=50^{\circ}$. The amplitude of the disturbances are very small beyond $\theta=120^{\circ}$. This implies that it is difficult to perturb the boundary layers near the leeward side using small roughness elements. When the generated vortices evolve downstream as shown in Figs. 15(b)-(e), they grow in amplitude and shift towards the leeward side. At $x=150 \mathrm{~mm}$, Fig. 15(e), about six vortices appear within the region $\theta=70^{\circ}$ to $160^{\circ}$ and no noticeable perturbations exist near the leeward plane $\theta>160^{\circ}$ or the windward plane $\theta<50^{\circ}$. For the line plots presented in Fig. 15, the crossflow vortices are evident by noting the individual peaks which are associated with a given crossflow vortex. The roughness elements for pattern 1 were placed in a ring at $x=50 \mathrm{~mm}$ and it is not obvious that the mean flow is affected until $x=100 \mathrm{~mm}$. For each successive downstream station shown in Fig. 15, the evolution of the crossflow vortices is seen and it is easy to follow the trajectory to see the migration of each vortex toward the leeward plane. We marked the peaks of an individual vortex with dots of a particular color (black, blue and red). For example, the vortex that originated near $x$ $=55 \mathrm{~mm}, \theta=43^{\circ}$ in Fig. 15 (a) drifted to $\theta=107^{\circ}$ at $x=150 \mathrm{~mm}$ (Fig. 15(e)). Later in this section, we will present the trajectories, stream-wise and span-wise wavelengths, and the growth of the amplitudes of the roughness pattern 1 vortices.

The variation of the maximum axial velocity perturbations along rays $\theta=30^{\circ}, 60^{\circ}, 90^{\circ}$ and $120^{\circ}$ is shown in Figs. 16(a)-(d). These figures give the wavelength and the amplitude variation of the vortices along different rays. Since the vortices propagate from the windward to the leeward side, the disturbances are confined to a finite region along each ray near the windward side and then spread along the entire ray near the leeward side. Along the $30^{\circ}$ ray, no disturbance growth occurs along the axial direction. Along the $60^{\circ}$ and $90^{\circ}$ rays, the perturbations grow for a certain axial distance and then decay further downstream. Along the $120^{\circ}$ ray, the perturbations appear downstream of the roughness elements and continuously grow along the axial direction.

Similarly, the quantitative description of the crossflow vortices formed downstream of the roughness elements for pattern 2 are displayed in Figs. 17 and 18. However, since the roughness elements are distributed along a cone ray, it is not as easy to track the trajectory development in azimuthal line plots, especially since it appears that some of the vortex trajectories split in two as they develop downstream. In Fig. 17, we assigned a vortex number associated with the roughness element from which the individual vortex originates. At $x=55 \mathrm{~mm}$, we see five vortices originating from the first five roughness elements located at $x=15,25,35,45$, and $55 \mathrm{~mm}$. At the next downstream station, $x=75 \mathrm{~mm}$, we see another vortex forming between the second and the third vortex. At $x=125$ $\mathrm{mm}$, another vortex forms between the third and the fourth vortices. It is also seen that the vortices 1-3 and 4-6 have different evolution characteristics. Also note that the amplitudes vary nonuniformly in the azimuthal direction. This may be due to the different initial amplitudes of the vortices, which may be the result of the potentially non-optimal wavelength we used in the simulation.

The quantitative crossflow vortex characteristics for roughness pattern 1 are presented in Figs. 19 and 20. The trajectories for ten vortices along with trajectory identification labels are shown in Fig. 19(a). First, note that the vortices are formed from the roughness elements located between $\theta=20^{\circ}$ to $110^{\circ}$. The trajectories from the lower five roughness elements move gradually up the side of the cone and do not appear to be experiencing any significant crowding effects from neighboring crossflow vortices. The five upper roughness elements produce vortices, which experience significant crowding as they move downstream. The azimuthal spacing between the vortices and their variation along the axis of the cone are presented in Figs. 20(a) and (b). Figure 20(a) presents the azimuthal wavelength in degrees while Fig. 20(b) displays the corresponding results in the form of a wavenumber. As expected, the initial wavelength of the vortices is approximately the same as the distance between the roughness elements, which is $9^{\circ}$. The downstream variation of the wavenumber is different for each vortex. The distance between the vortices in the region closer to the windward side, vortices 1 to 4 , first decreases, then reaches a plateau before increasing further downstream. For example, the wavenumber for the vortex 3 decreases from $m=40$ to $m=$ 26 at $x=120 \mathrm{~mm}$ and then gradually increases to $m=35$ at $x=200 \mathrm{~mm}$. The wavenumbers of the vortices in the region closer to the leeward side continue to increase. The wavenumber for the vortex 8 continues to increase from $m=40$ to $m=70$ at $x=150 \mathrm{~mm}$. The important and relevant question of how the system is selecting this wavenumber variation is not answered in this work. Further analysis is needed to be able to theoretically predict the different wavenumber variations observed in these simulations. Finally, Fig. 19(b) shows the maximum $u$-velocity perturbation growth for roughness pattern 1 along selected vortex trajectories. The trajectory identified as number five appears to be the most amplified as compared to the other four trajectories shown in this figure. Trajectory number five has the greatest portion of its path length in the highest crossflow region (see Fig. 8) as compared with the other four trajectories shown in Fig. 19(b). 
The next question addressed is how does the computed growth, and the azimuthal and axial wavenumbers of the vortices compare with the linear stability results? These results are presented in Figs. 21(a-c) and 22(a-b). In the stability computations, we use the information presented in Figs. 19(a) and 20(b). We select one vortex, for example vortex 5, and use the wavenumber variation for that vortex shown in Fig. 20(b) to calculate the growth rate for that vortex from the stability theory along its trajectory. Figures 21(a-c) show the growth rates computed from linear stability theory for different azimuthal wavenumbers at three axial stations $x=55,100$ and $150 \mathrm{~mm}$. We then placed the growth rates obtained for the three vortices 3,5 and 8 at these stations in the figures. At the station $x=55 \mathrm{~mm}$, the growth rates of the vortices are closer to the maximum growth rates at this station. Moving further downstream to stations $x=100$ and $150 \mathrm{~mm}$, only the growth rates for vortex 5 remain close to the predicted maximum growth rates at each station. We integrated the growth rate along its trajectory to compute the amplitude variation. The results are shown in Fig. 22(a) for the vortices 3 and 5. The agreement between the results obtained from the simulation and from the parallel linear stability theory is remarkably good. Comparisons between the calculated axial wavelengths obtained from these methods are displayed in Fig. 22(b) and the agreement is very good. The wavelengths gradually increase along the cone for all the vortices. The wavelength for the vortex 5 increased from $11 \mathrm{~mm}$ at $x=60 \mathrm{~mm}$ to $25 \mathrm{~mm}$ at $x=170 \mathrm{~mm}$, and the wavelength for vortex 3 increased from $15 \mathrm{~mm}$ to $30 \mathrm{~mm}$ over the same axial distance.

\section{Discussion and Conclusions}

We investigated the stability, transition onset, and generation of crossflow vortices in a three-dimensional hypersonic boundary layer over a sharp-tipped, $7^{\circ}$ half-angle cone at an angle of attack of $6.0^{\circ}$. Mean flow computations showed that the boundary layer near the leeward plane is separated. The boundary-layer thickness along the leeward plane is about 10 times thicker than that along the windward plane. The boundary-layer profiles are self-similar along each ray except near the leeward planes. Simulations also revealed that the crossflow reaches a maximum just above the sides of the cone and becomes weaker near the leeward and the windward sides. However, substantial crossflow velocity still exists up to $\theta=165^{\circ}$ from the windward plane.

The linear stability results showed that the stationary disturbances are more stable than the traveling disturbances. The growth rates of the traveling disturbances in the middle of the cone are about 10 to 20 percent higher than for the stationary disturbances. This difference increases in the region closer to the windward side. The azimuthal wavenumbers for the unstable traveling disturbances are in the range of $20-50$ while those for the stationary disturbances are in the range of $30-70$. The N-Factor computations predicted that transition would occur farther upstream near the sides of the cone than along the windward and the leeward planes. The boundary layers near the leeward plane are not as stable as the boundary layers near the windward plane. Transition onset is predicted to occur farther downstream for the stationary disturbances than for traveling disturbances. However, these relative locations are dependent on the free-stream disturbance levels and the roughness distribution on the surface. The N-Factor traces indicated that transition is caused by disturbances originating from regions closer to the windward side of the cone.

We studied the generation and evolution of stationary crossflow vortices by performing simulations with two types of roughness patterns placed on the surface of the cone. The first pattern consists of small periodic roughness elements placed around the cone at a fixed axial location closer to the tip. The second pattern consists of small roughness elements of constant spacing placed along a ray closer to the windward side. The direct numerical simulation shows that crossflow vortices originating from these roughness elements located near the windward side grow due to the crossflow instability and migrate towards the leeward side of the cone. No significant perturbations are induced by the roughness elements on the leeward side of the cone, $90^{\circ} \leq \theta \leq 180^{\circ}$. The maximum perturbation amplitude formed a smooth arc shape for pattern 1 with the largest amplitude occurring in a region extending forward on the sides of the cone. The azimuthal wavenumber of the generated vortices are initially the same as the input wavenumber of the roughness pattern. The wavenumber variation of the vortices on the windward side of the cone initially decreases, plateaus, and then may increase further downstream as they migrate through the maximum crossflow region. The wavenumbers of the vortices that lay in the leeward crossflow region continuously increase. The movement of the roughness elements to locations different from this study may affect the conclusions drawn here. We have to relate the growth rates and the wavelengths obtained from the linear stability theory to the perturbations field obtained from the simulation. The question of how to decompose the computed flow field into normal-mode type disturbances in a three-dimensional flow will need to be analyzed in the future. 


\section{References}

${ }^{1}$ Mack, M.L., "Boundary Layer Stability Theory,” JPL Report No. 900-277 Rev. A, Pasadena, CA, Nov. 1969.

${ }^{2}$ Kendall, J.M., "Wind Tunnel Experiments Relating to Supersonic and Hypersonic Boundary-Layer Transition," AIAA J., Vol. 13, No. 3, 1975, pp. 290-299.

${ }^{3}$ Stetson, K.F., and Kimmel, R.L., “On Hypersonic Boundary-Layer Stability,” AIAA Paper 92-0737, January 1992.

${ }^{4}$ Maslov, A.A., Shiplyuk, A.N., Sidorenko, A.A and Arnal, D., "Leading Edge Receptivity of a Hypersonic Boundary layer on a Flat Plate," J. Fluid Mech., Vol. 426, 2001, pp. 73-94.

${ }^{5}$ Fedorov, A.V., and Khokhlov, A.P., " Excitation of Unstable Modes in a Supersonic Boundary Layer by Acoustic Waves," Fluid Dynamics, Vol. 26, No. 4, July 1991, pp. 531-537.

${ }^{6}$ Fedorov, A.V., "Receptivity of a High-Speed Boundary Layer to Acoustic Disturbances," Journal of Fluid Mechanics, Vol. 491, 2003, pp. 101-129.

${ }^{7}$ Ma, Y. and Zhong, X., "Receptivity of a Supersonic Boundary Layer over a Flat Plate. Part 1. Wave Structures and Interactions," Journal of Fluid Mechanics, Vol. 488, 2003, pp. 31-78.

${ }^{8} \mathrm{Ma}$, Y. and Zhong, X., "Receptivity of a Supersonic Boundary Layer over a Flat Plate. Part 2. Receptivity to Freestream Sound," Journal of Fluid Mechanics, Vol. 488, 2003, pp. 79-121.

${ }^{9}$ Malik, M.R., "Boundary-Layer Transition Prediction Toolkit,", AIAA paper 1997-1904.

${ }^{10}$ Malik, M.R., and Balakumar, P., "Instability and Transition in Three-Dimensional Supersonic Boundary Layers," AIAA Paper 1992-5049, 1992.

${ }^{11}$ Kara, K., Balakumar, P. and Kandil, O.A., "Receptivity of Hypersonic Boundary Layers Due to Acoustic Disturbances over Blunt Cone," 45th Aerospace Sciences Meeting and Exhibit, Reno, NV, AIAA 2007-0945.

${ }^{12}$ Kara, K., Balakumar, P. and Kandil, O.A., "Effects of Nose Bluntness on Stability of Hypersonic Boundary Layers over a Blunt Cone," 37th Fluid Dynamics Conference \& Exhibit, Miami, FL, AIAA 2007-4492.

${ }^{13}$ Kara, K., Balakumar, P. and Kandil, O.A., "Effects of Wall Cooling on Hypersonic Boundary Layer Receptivity over a Cone," 38th Fluid Dynamics Conference \& Exhibit, Seattle, WA, AIAA 2008-3734.

${ }^{14}$ Balakumar, P., and Kegerise, M. A., "Receptivity of Hypersonic Boundary Layers over Straight and Flared Cones," AIAA Paper 2010-1545, January 2010.

${ }^{15}$ Potter, J.L., Boundary Layer Transition on Supersonic Cones in an Aerobalistic Range, AIAA Journal, Vol. 13, No. 3,270-277, 1975 .

${ }^{16}$ Krogmann, P., "An Experimental Study of Boundary Layer Transition on a Slender Cone at Mach 5," AGARD Conference Proceedings N0.224, Laminar Turbulent Transition, May 1977.

${ }^{17}$ Stetson, K.F., "Effect of Bluntness and Angle of Attack on Boundary Layer Transition on Cones and Biconic Configurations", $17^{\text {th }}$ Aerospace Sciences Meeting, New Orleans, LA, January 15-17, 1979. AIAA 79-0269.

${ }^{18}$ Stetson, K.F., "M = 6 Wind Tunnel Experiments of Boundary Layer Transition on a Cone at Angle of Attack", $14^{\text {th }}$ Fluid and Plasma Dynamics Conference, Palo Alto, CA, June 23-25, 1981. AIAA 81-1226.

${ }^{19}$ King, R.A., "Three-dimensional boundary layer transition on a cone at Mach 3.5", Experiments in Fluids, Vol. 13, pp. 305-314, 1992.

${ }^{20}$ Stetson, K.F., Thompson, E.R., Donaldson, J.C., and Siler, L.G., "Laminar Boundary Layer Stability Experiments on a Cone at Mach 8, Part 1: Sharp Cone," AIAA 22 ${ }^{\text {nd }}$ Aerospace Sciences Meeting, Reno, Nevada, January 9-12, AIAA Paper 83-1761, 1983.

${ }^{21}$ Stetson, K.F., Thompson, E.R., Donaldson, J.C., and Siler, L.G., "Laminar Boundary Layer Stability Experiments on a Cone at Mach 8, Part 2: Blunt Cone," AIAA 22 ${ }^{\text {nd }}$ Aerospace Sciences Meeting, Reno, Nevada, January 9-12, AIAA Paper 84-0006, 1984.

${ }^{22}$ Stetson, K.F., Thompson, E.R., Donaldson, J.C., and Siler, L.G., "Laminar Boundary Layer Stability Experiments on a Cone at Mach 8, Part 3: Sharp Cone at Angle of Attack," AIAA $22^{\text {nd }}$ Aerospace Sciences Meeting, Reno, Nevada, January 9-12, AIAA Paper 85-0492, 1985.

${ }^{23}$ Stetson, K.F., Thompson, E.R., Donaldson, J.C., and Siler, L.G., "Laminar Boundary Layer Stability Experiments on a Cone at Mach 8, Part 4:On Unit Reynolds Number and Environmental Effects," AIAA $22^{\text {nd }}$ Aerospace Sciences Meeting, Reno, Nevada, January 9-12, AIAA Paper 86-1087, 1986.

${ }^{24}$ Stetson, K.F., Thompson, E.R., Donaldson, J.C., and Siler, L.G., "Laminar Boundary Layer Stability Experiments on a Cone at Mach 8, Part 5: Tests with cooled Model," AIAA 22 ${ }^{\text {nd }}$ Aerospace Sciences Meeting, Reno, Nevada, January 9-12, AIAA Paper 89-1895, 1989.

${ }^{25}$ Blanchard, A.E., An investigation of Wall-Cooling Effects on Hypersonic Boundary-Layer Stability in a Quiet Wind Tunnel, Ph.D. Dissertation, Old Dominion University, Dept. of Mechanical Engineering, Norfolk, VA, December, 1995.

${ }^{26}$ Lachowicz, J.T., Chokani, N., and Wilkinson, S.P., "Boundary-Layer Stability Measurements in a Hypersonic Quiet Tunnel”, AIAA Journal, Vol. 34, No. 12, December 1996.

${ }^{27}$ Dogget, G.P., and Chokani, N., "Effects of Angle of Attack on Hypersonic Boundary-Layer Stability," AIAA Journal, Vol. 35, No. 3, March 1997, pp. 464-470.

${ }^{28}$ Wheaton, B., Juliano, T.J., Berridge, D.C., Chou, A., Gilbert, P.L., Casper, K.M., Steen, L.E., Schneider, S., and Johnson, H.B., "Instability and Transition Measurements in the Mach 6 Quiet Tunnel," AIAA Paper 2009-3559, June 2009.

${ }^{29}$ Casper, K.M., Beresh, S.J., Henfling, J.F., Spillers, R.W., Pruett, B., and Schneider, S.P., "Hypersonic Wind-Tunnel Measurements of Boundary-Layer Pressure Fluctuations,” AIAA Paper 2009-4054, Jun 2009.

\section{0 of 21}

American Institute of Aeronautics and Astronautics AIAA-2010-4718 


\footnotetext{
${ }^{30}$ Juliano, T.J., Swanson, E.O., and Schneider, S.P., "Transition Research and Improved Performance in the Boeing/AFOSR Mach-6 Quiet Tunnel", 45 ${ }^{\text {th }}$ Aerospace and Science Meeting and Exhibit, Reno, NV, January 2007. AIAA 2007-535.

${ }^{31}$ Swanson, E.O., and Schneider, S.P., "Boundary-Layer Transition on Cones at Angle of Attack in a Mach-6 Quiet Tunnel," $48^{\text {th }}$ AIAA Aerosciences Meeting including the New Horizons Forum and Aerospace Exposition, January 2010. AIAA 2010-1062.

${ }^{32}$ Kroonenberg, A., Radespiel, R., Candler, G., and Estorf, M., "Infrared measurements of boundary-layer transition on an inclined cone at Mach 6," 48 ${ }^{\text {th }}$ AIAA Aerosciences Meeting including the New Horizons Forum and Aerospace Exposition, January 2010. AIAA 2010-1063.

${ }^{33}$ Balakumar, P., and Reed, H. L., "Stability of Three-Dimensional Supersonic Boundary Layers," Physics of Fluids, Vol. 3, No. 4, April 1991, pp. 617-632.

${ }^{34}$ Balakumar, P., "Stability of Supersonic Boundary Layers on a Cone at an Angle of Attack," $39^{\text {th }}$ Fluid Dynamics Conference and Exhibit, June 22-25, 2009, San Antonio, Texas. AIAA 2009-3555.

${ }^{35}$ Malik, M.R., "eMalik3d: An $\mathrm{e}^{\mathrm{N}}$ Code for Three-Dimensional Flow over Finite-Swept Wings", High Technology Report No. HTC-9502, 1995.

${ }^{36}$ P. Balakumar, "Receptivity of Supersonic Boundary Layers Due To Acoustic Disturbances Over Blunt Cones," AIAA Paper 2007-4491, 2007.

${ }^{37}$ Shu, Chi-Wang, “ Essentially Non-Oscillatory and Weighted Essentially Non-Oscillatory Schemes for Hyperbolic Conservation Laws," NASA/CR-97-206253 and ICASE Report N0. 97-6

${ }^{38}$ Atkins, H.L., "High-Order ENO Methods for the Unsteady Compressible Navier-Stokes Equations," AIAA Paper 91 1557, 1991.

${ }^{39}$ Balakumar, P., Zhao, H., and Atkins, H., "Stability of Hypersonic Boundary-layers Over a Compression Corner," AIAA Paper 2002-2848, 2002.

${ }^{40}$ Malik, M.R., and Balakumar, P., "Instability and Transition in Three-Dimensional Supersonic Boundary Layers," AIAA Paper 1992-5049, 1992.
} 


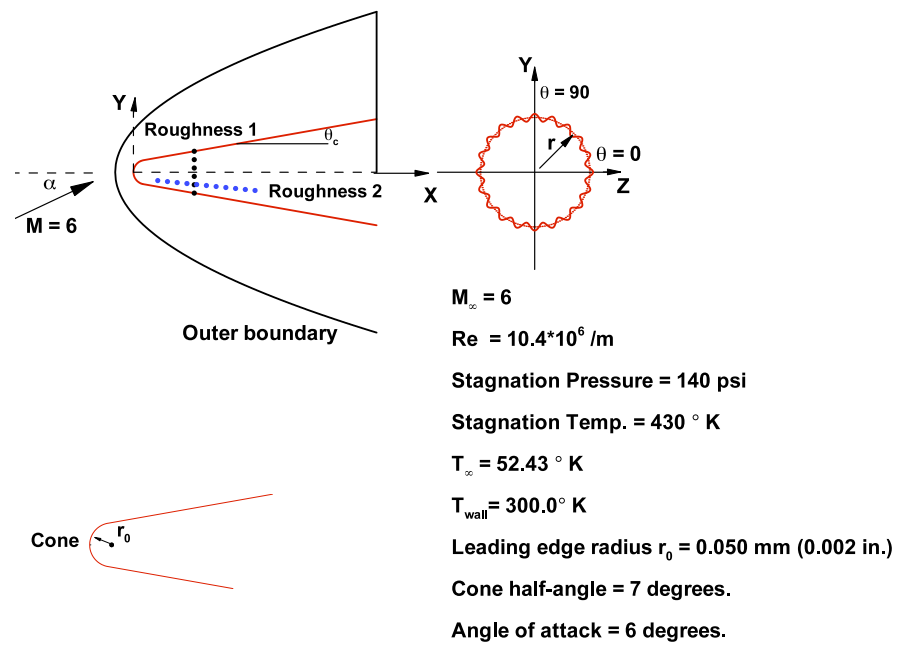

Figure 1. Schematic diagram representing the computational model.

(a)



(b)

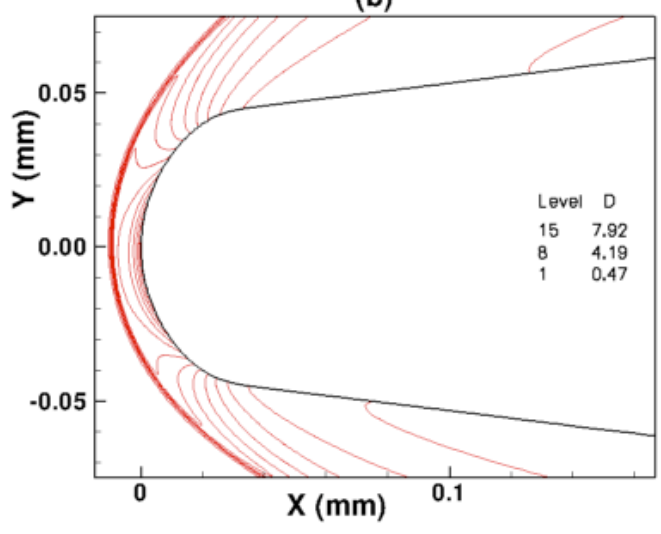

Figure 2. Contours of the density for flow over a $7^{\circ}$ half-angle cone with a blunted nose tip at $\mathrm{M}=6.0$ and at an angle of attack of 6.0 degrees, $\operatorname{Re}=10.4 * 10^{6} / \mathrm{m}$.

(a) $\mathrm{x}=9 \mathrm{~mm}$

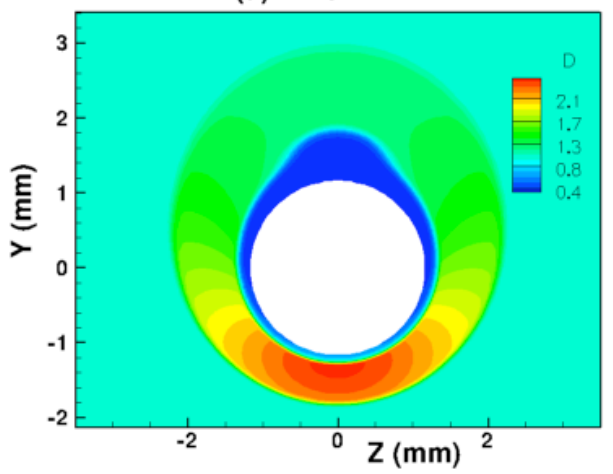

(b) $x=330 \mathrm{~mm}$

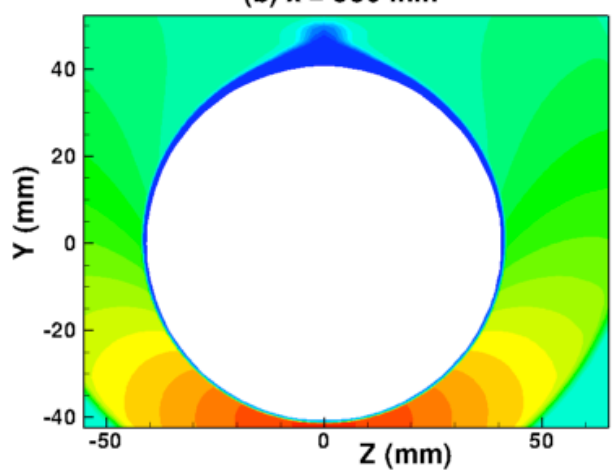

Figure 3. Contours of the density in the cross-sectional planes at different axial locations. 
(a) windward side

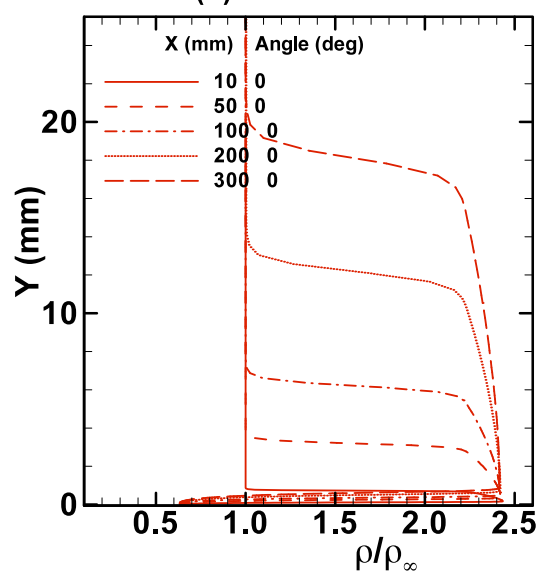

(b)

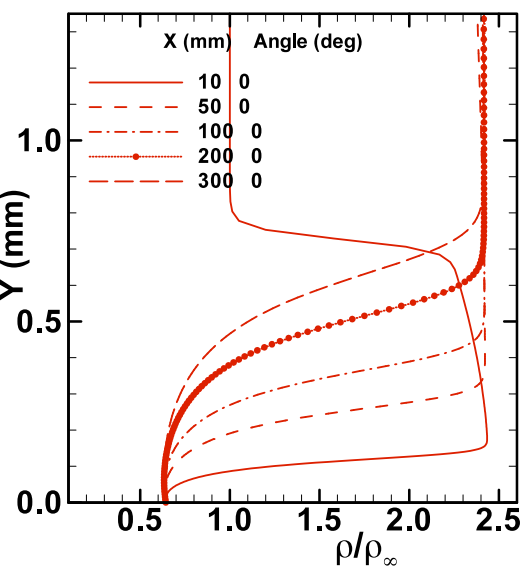

(c)

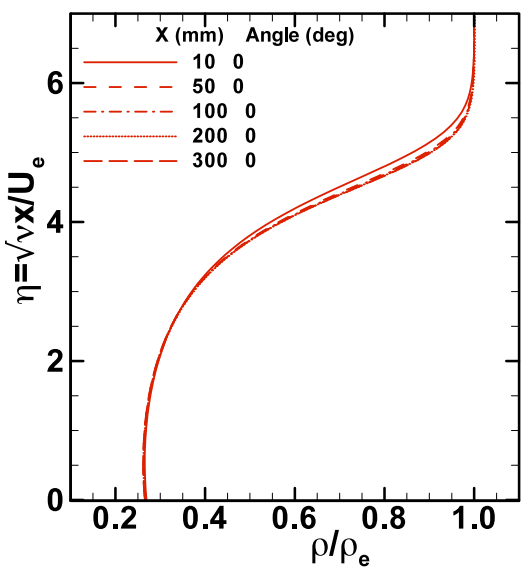

Figure 4. Density profiles along the windward side, $\theta=0^{\circ}$, at different axial locations.

(a) azimuthal angle $90 \mathrm{deg}$



(b)

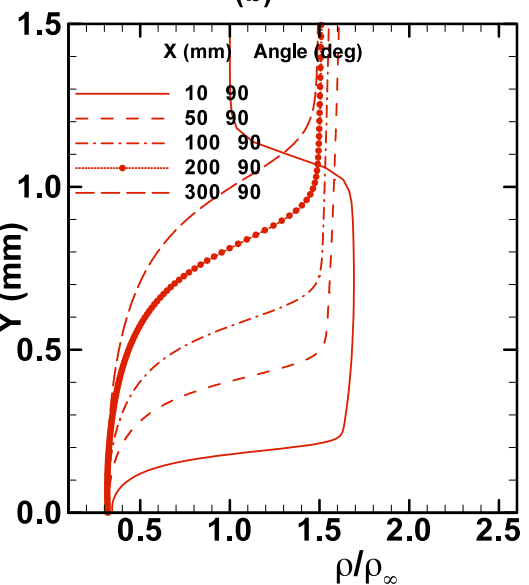

(c)

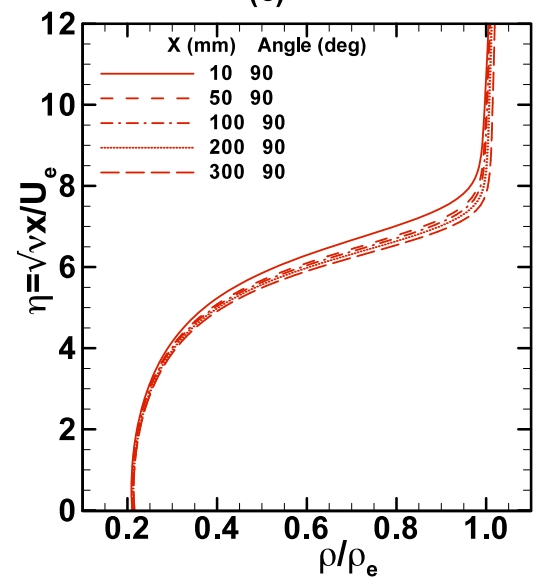

Figure 5. Density profiles along the azimuthal direction of $\theta=90^{\circ}$ at different axial locations.

(a) leeward side

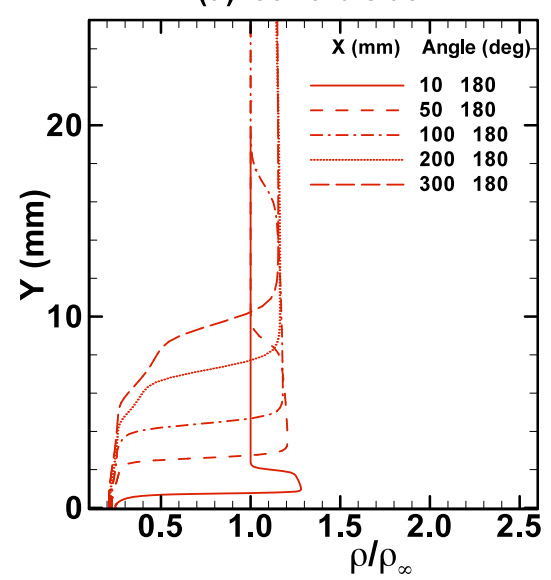

(b)

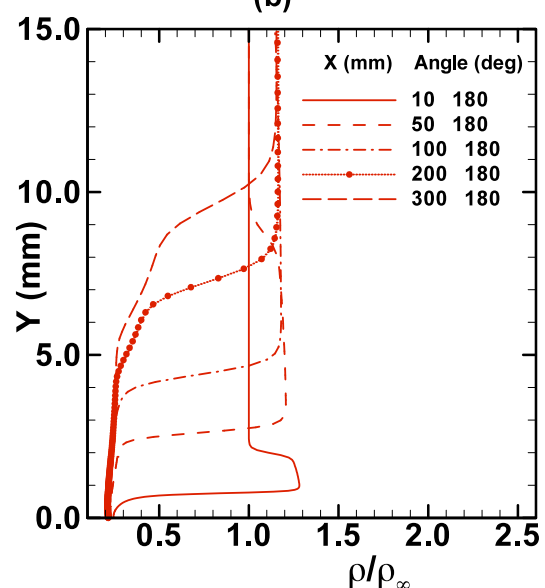

(c)

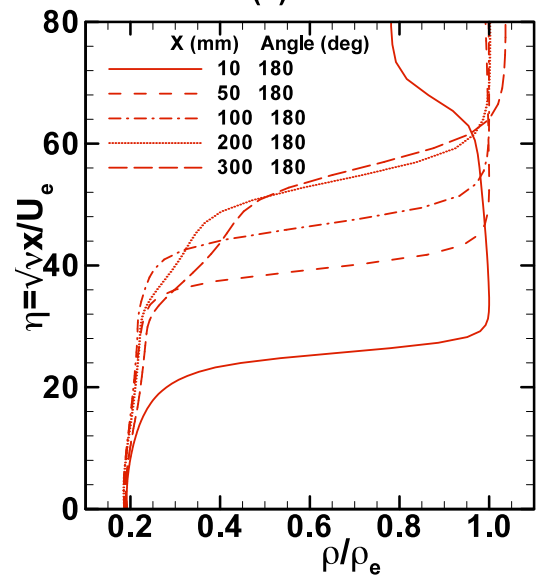

Figure 6. Density profiles along the leeward side, $\theta=180^{\circ}$, at different axial locations. 
(a)



(b)

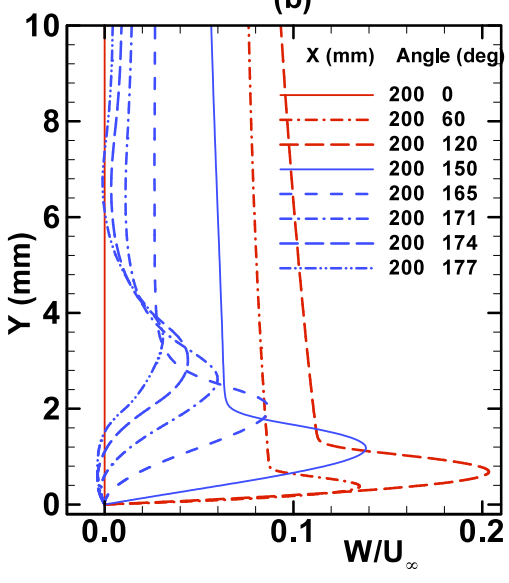

(c)

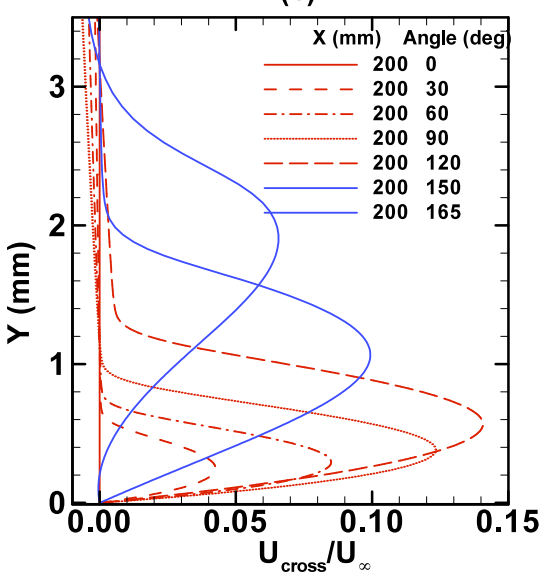

Figure 7. Boundary layer (a) stream-wise velocity (b) azimuthal velocity and (c) crossflow velocity profiles for different azimuthal angles at an axial location of $x=200 \mathrm{~mm}$.

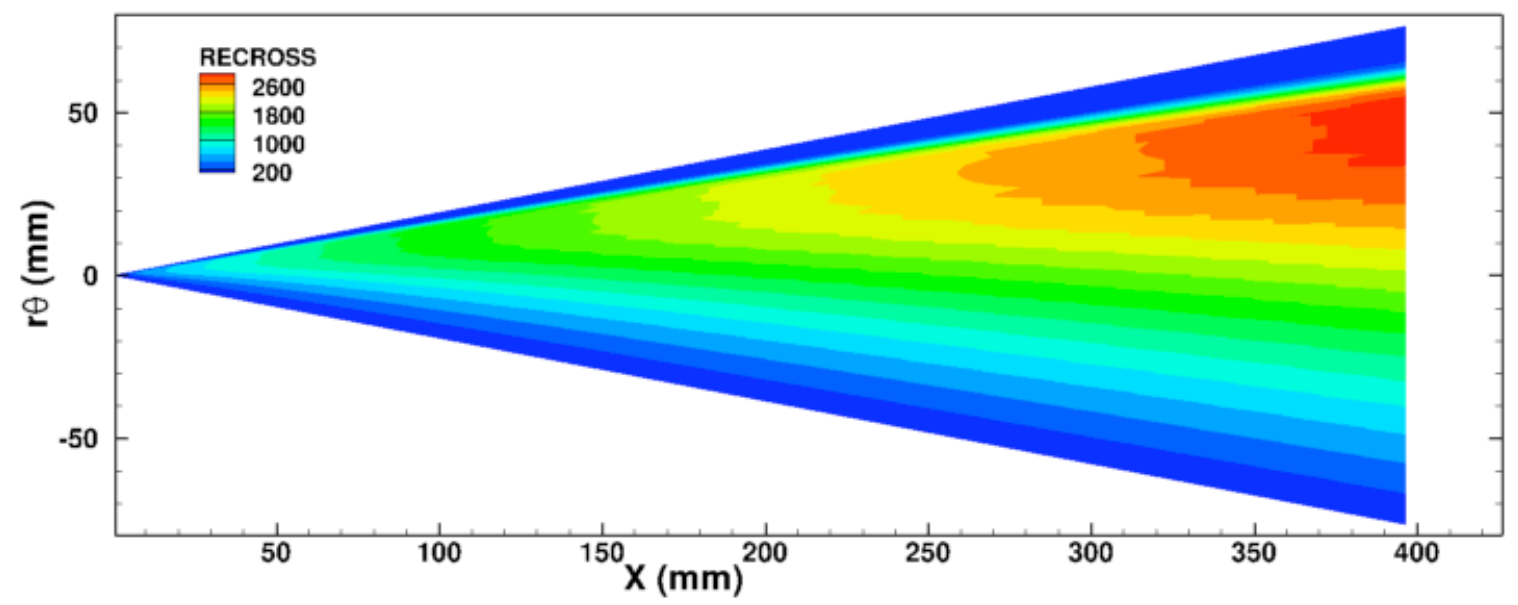

Figure 8. Crossflow Reynolds number for $M=6, \operatorname{Re}=10.4 * 10^{6} / \mathrm{m}$, Angle of attack $=6.0^{\circ}$. 

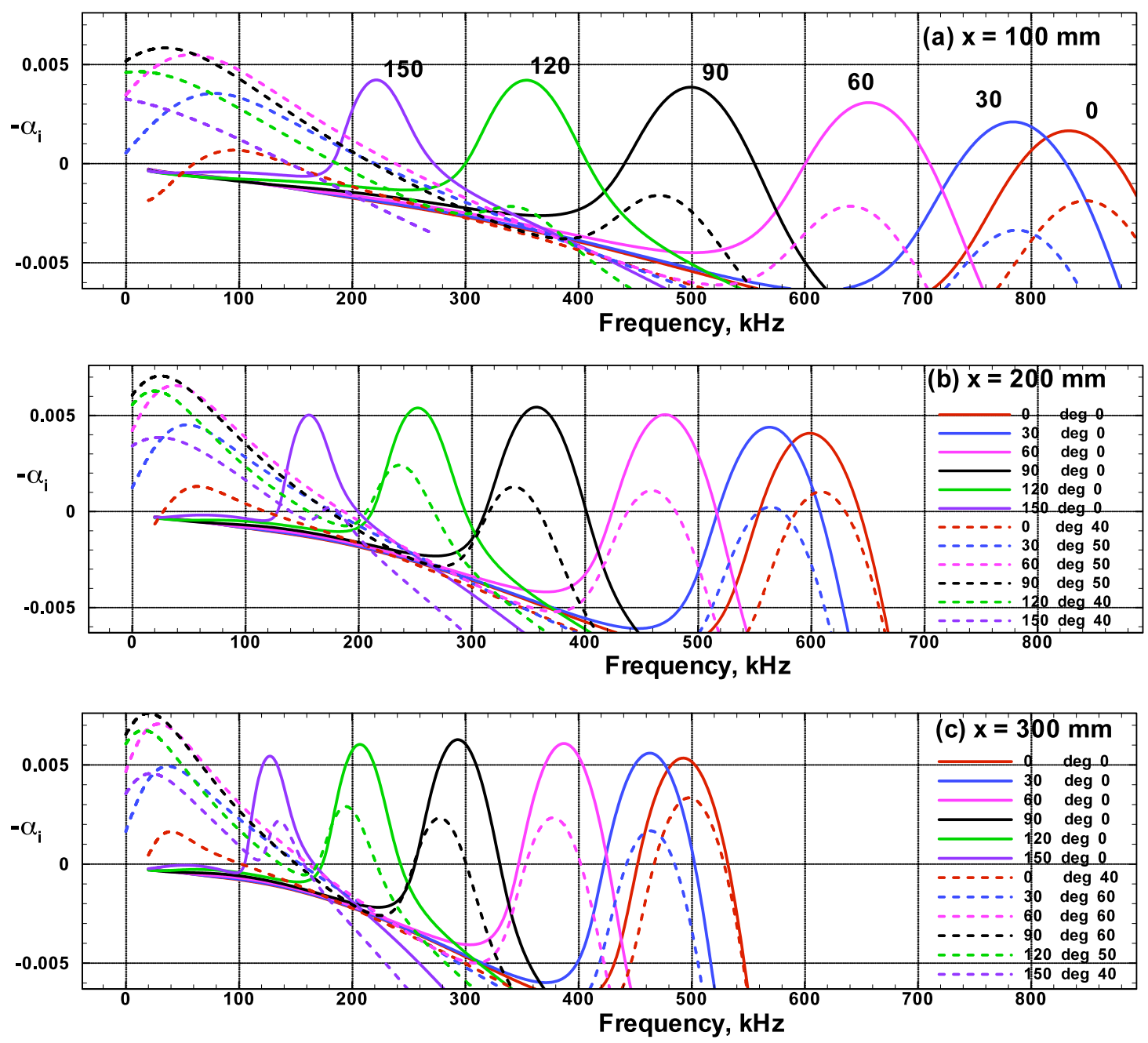

Figure 9. Growth rates for traveling and stationary disturbances at axial locations $x=100,200$, and $300 \mathrm{~mm}$ and $\operatorname{Re}=10.4 * 10^{6} / \mathrm{m}$. Legends in (b) and (c) list values for $\theta$ and $\mathrm{m}$.
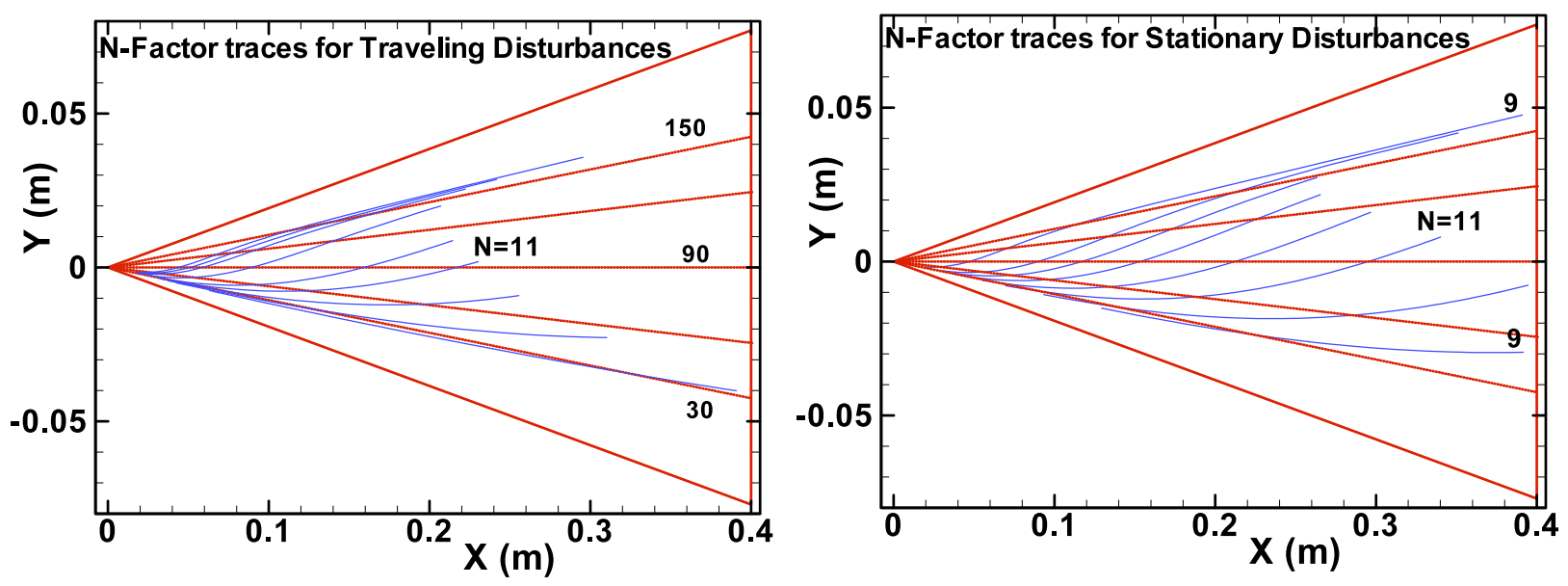

Figure 10. N-Factor traces for (a) traveling and (b) stationary disturbances. $R e=10.4 * 10^{6} / \mathrm{m}$. 

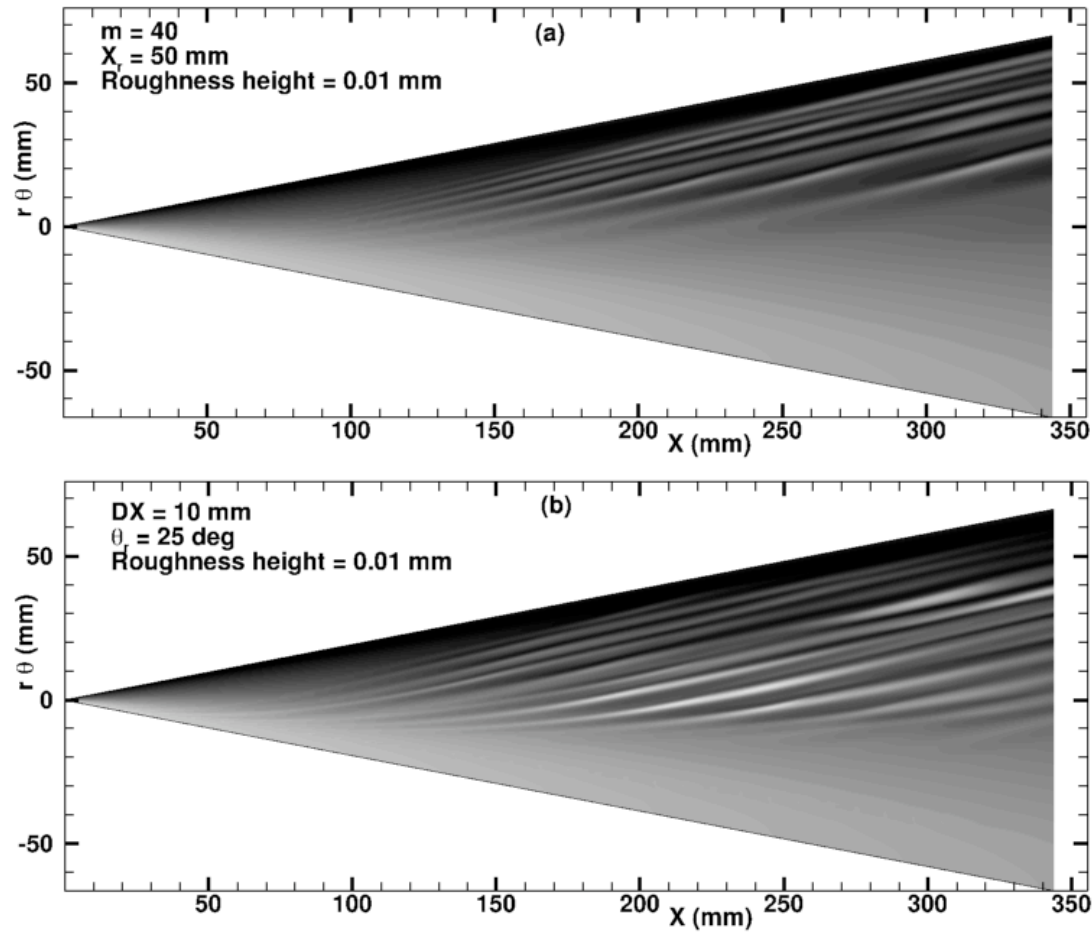

Figure 11. Contours of u-velocity, in the $x-r \theta$ planes. $R e=10.4 * 10^{6} / \mathrm{m},(a) \mathrm{m}=40, \mathrm{~h}=0.01 \mathrm{~mm}, \mathrm{x}_{\mathrm{r}}=50 \mathrm{~mm}$ (b) $\Delta \mathrm{x}=10 \mathrm{~mm}, \mathrm{~h}=0.01 \mathrm{~mm}, \theta_{\mathrm{r}}=25 \mathrm{deg}$.
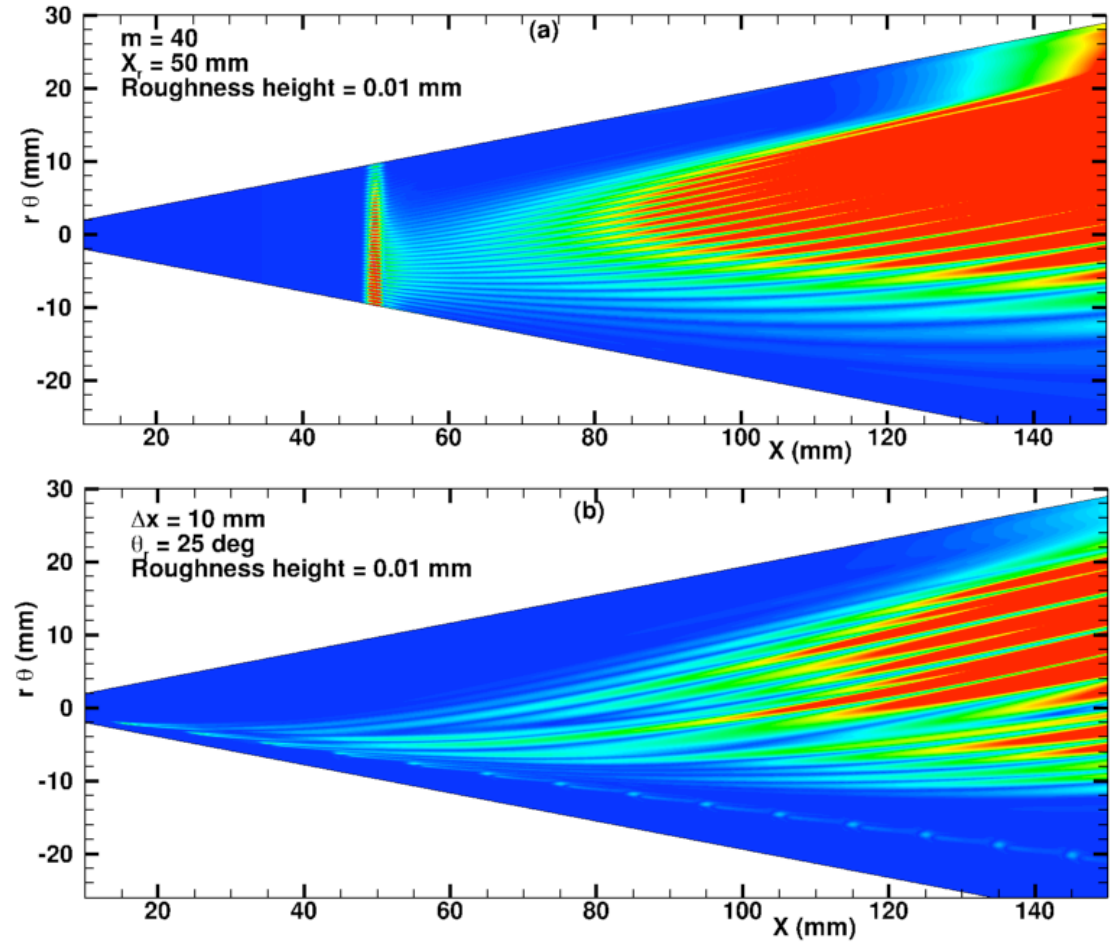

Figure 12. Contours of maximum u-velocity perturbations, in the $x-r \theta$ planes. $R e=10.4 * 10^{6} / \mathrm{m},(a) \mathrm{m}=40, \mathrm{~h}$ $=0.01 \mathrm{~mm}, x_{r}=50 \mathrm{~mm},(b) \Delta x=10 \mathrm{~mm}, h=0.01 \mathrm{~mm}, \theta_{\mathrm{r}}=25 \mathrm{deg}$. 
(d) $250 \mathrm{~mm}$

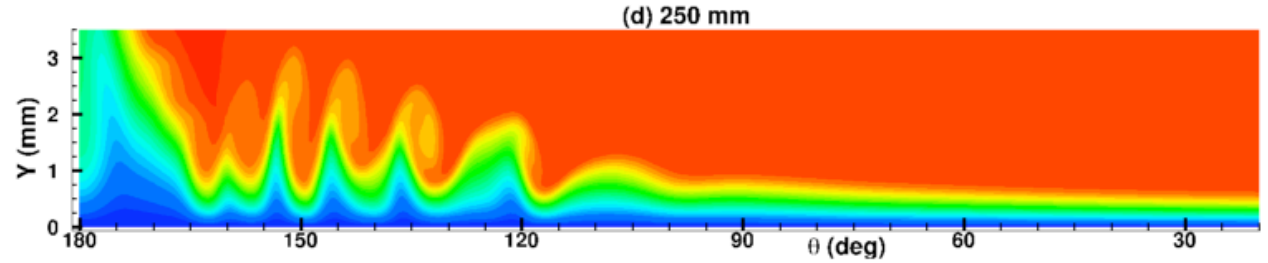

(c) $200 \mathrm{~mm}$

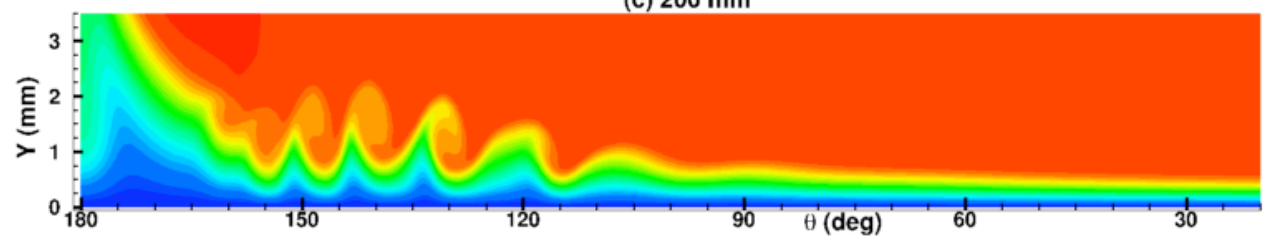

(b) $150 \mathrm{~mm}$

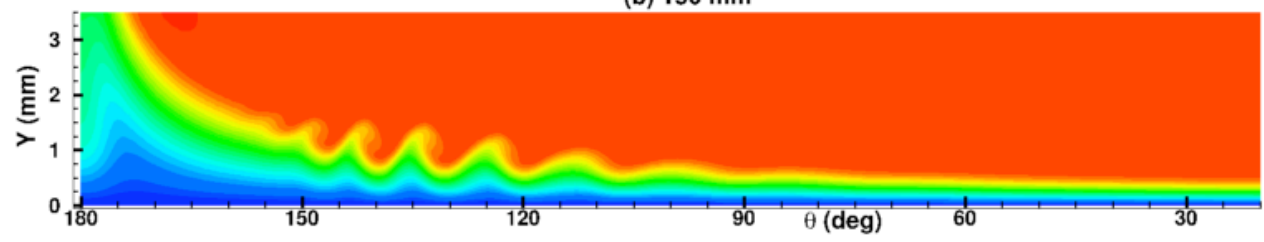

(a) $100 \mathrm{~mm}$

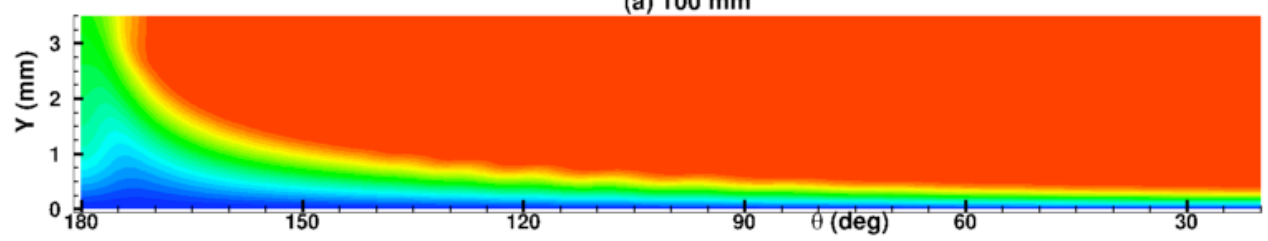

Figure 13. Contours of the u-velocity in the cross planes at different axial locations for roughness 1 pattern.

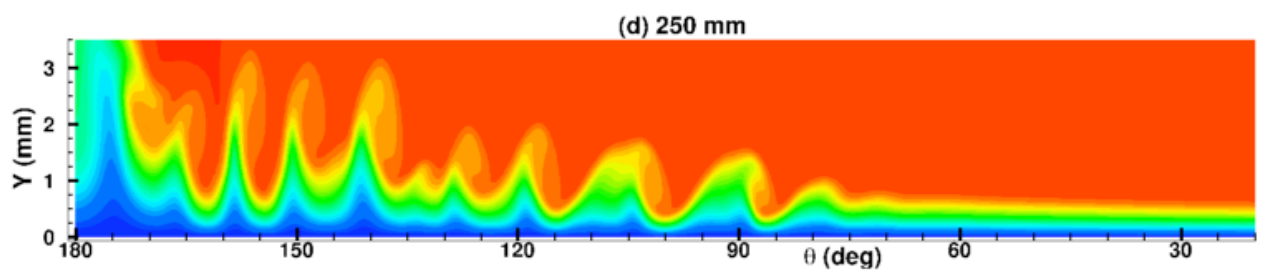

(c) $200 \mathrm{~mm}$

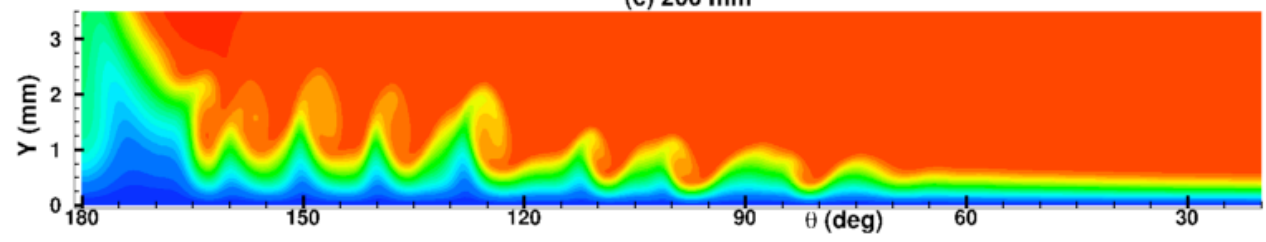

(b) $150 \mathrm{~mm}$

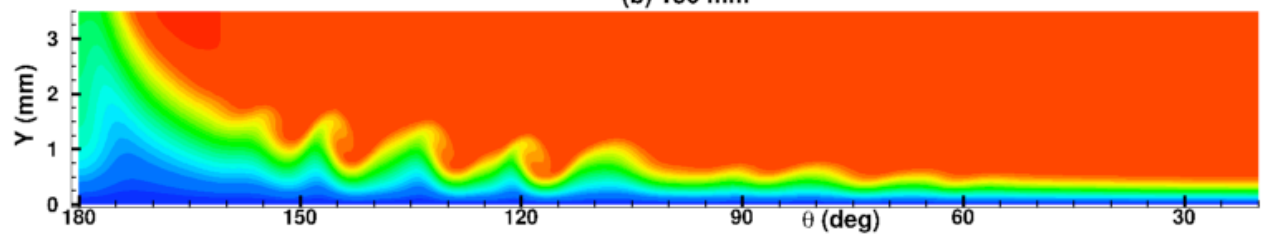

(a) $100 \mathrm{~mm}$

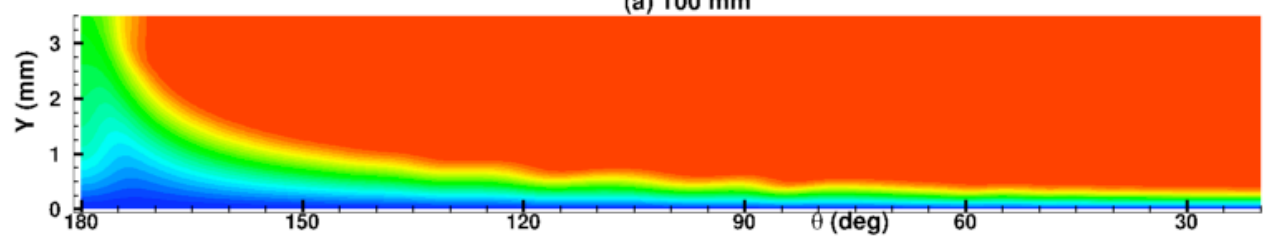

Figure 14. Contours of the u-velocity in the cross planes at different axial locations for roughness 2 pattern. 

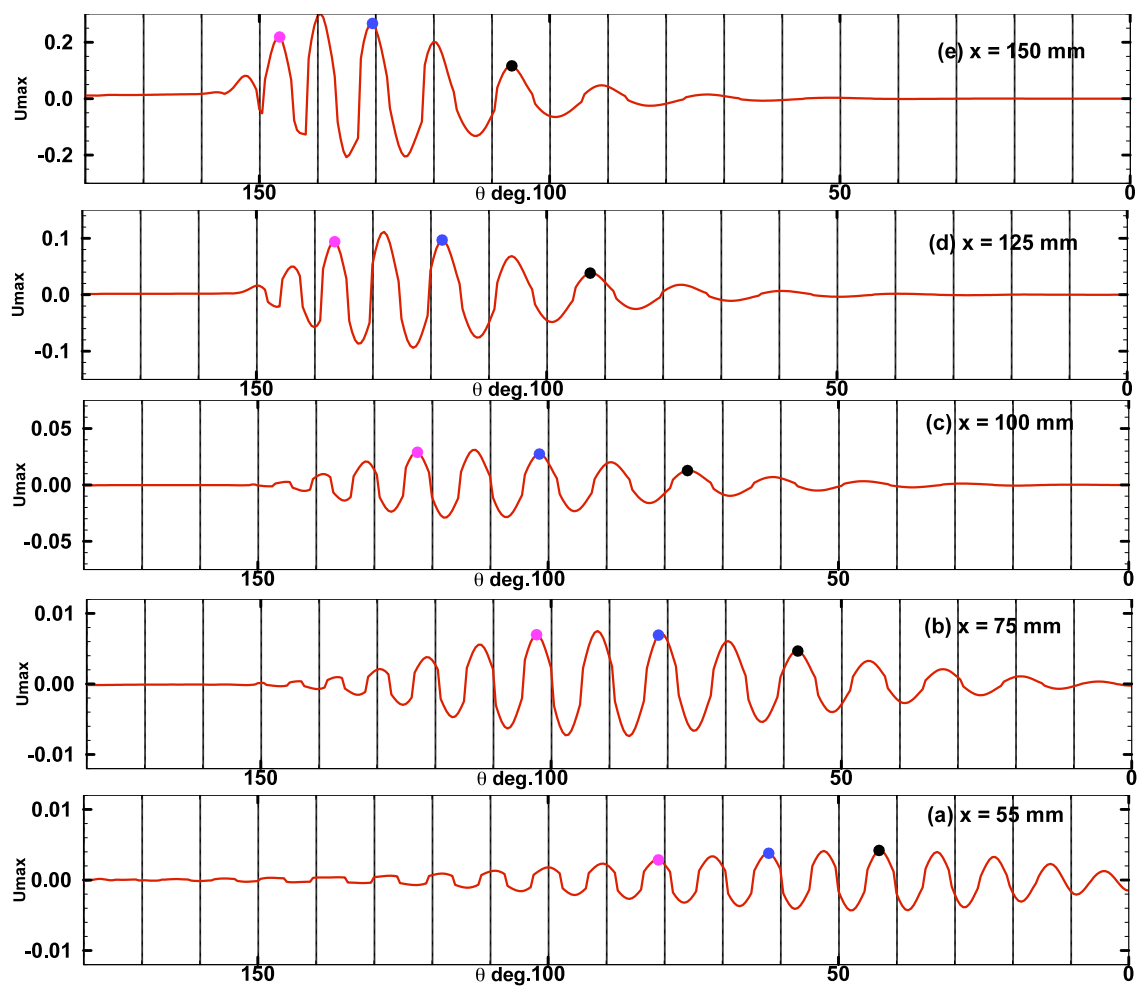

Figure 15. Perturbations of the maximum u-velocity downstream of the roughness 1 pattern at different axial locations.
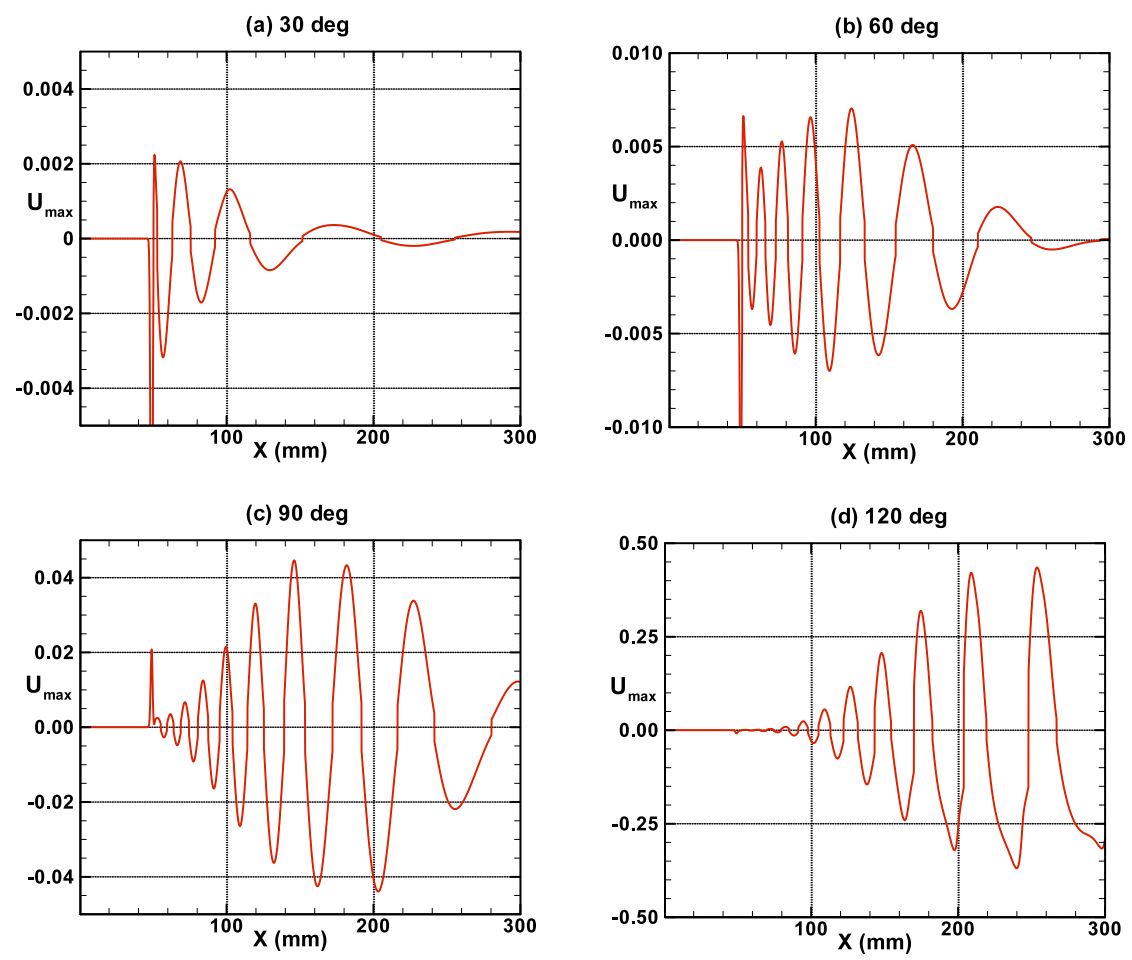

Figure 16. Perturbations of the maximum u-velocity in the axial direction along different rays for roughness 1. 


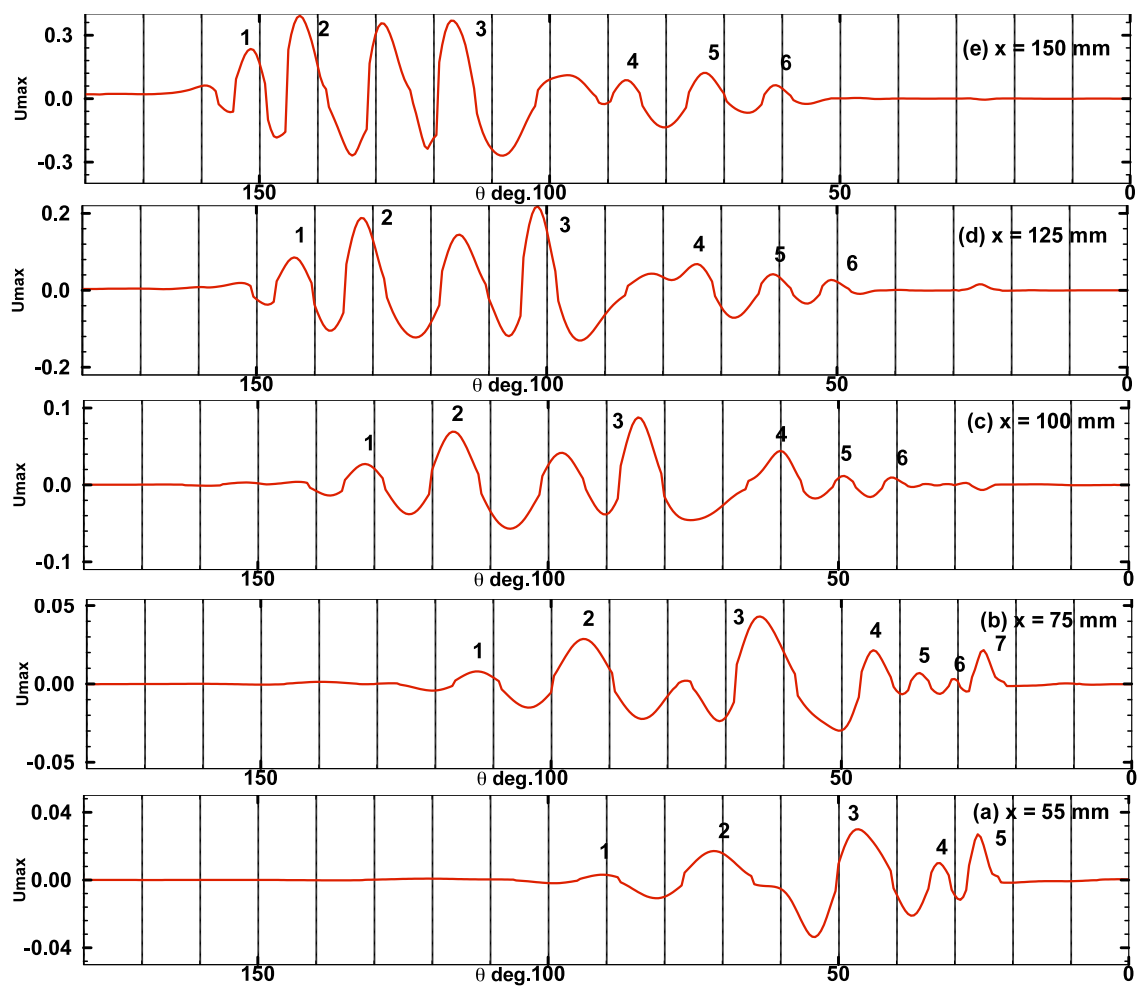

Figure 17. Perturbations of the maximum u-velocity downstream of the roughness 2 pattern at different axial locations.

(a) 30 deg

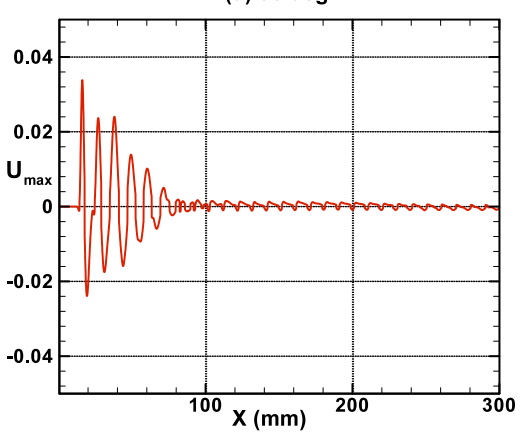

(c) $90 \mathrm{deg}$



(b) 60 deg

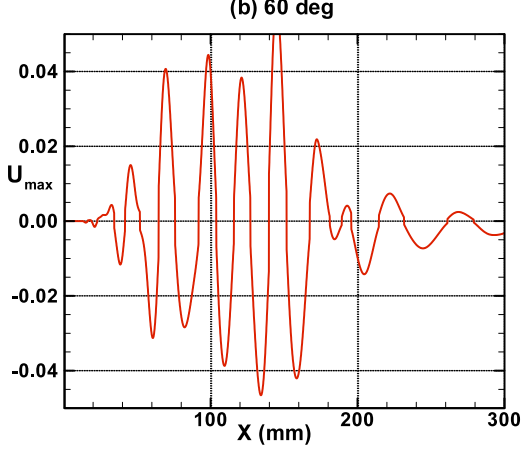

(d) $120 \mathrm{deg}$

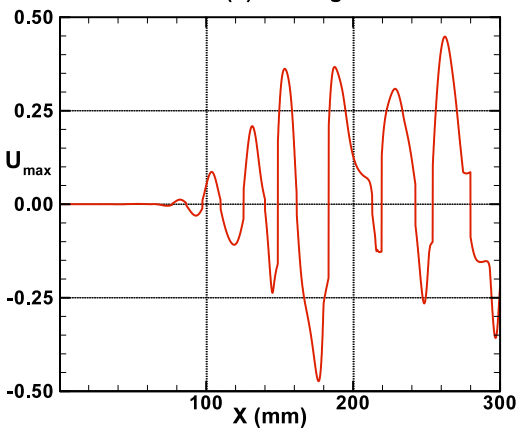

Figure 18. Perturbations of the maximum u-velocity in the axial direction along different rays for roughness 2. 

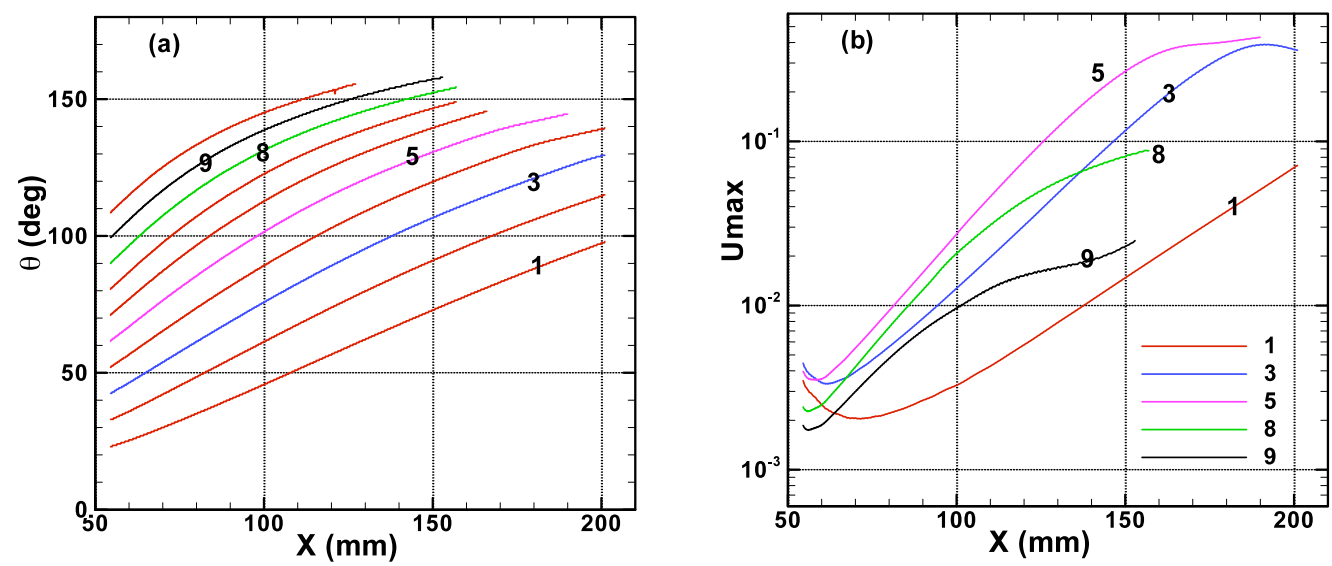

Figure 19. Disturbance vortex (a) trajectories and (b) velocity fluctuation growth for roughness 1 pattern at different axial locations (up to saturation levels).
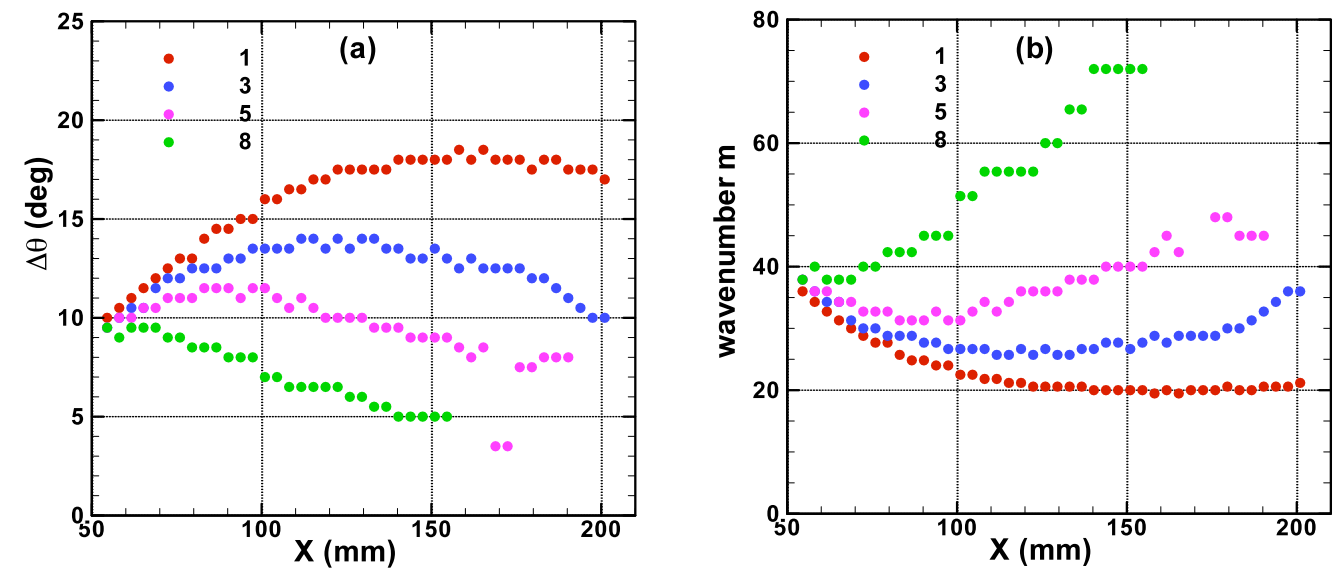

Figure 20. Disturbance vortices (a) azimuthal spacing and (b) wavenumber variation for roughness 1 pattern at different axial locations (up to saturation levels).


Figure 21. Growth rates for stationary disturbances at axial locations $x=55$, 100, and $150 \mathrm{~mm}$. 

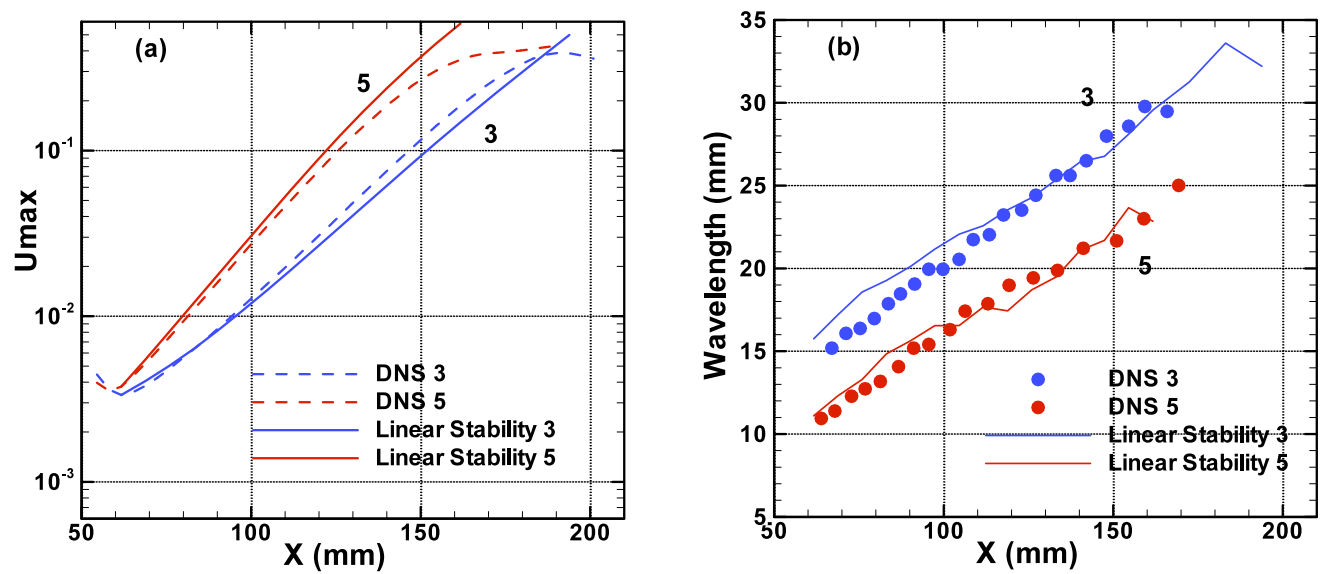

Figure 22. Comparisons of the amplitude and wavelengths computed from DNS and linear stability. 